\title{
Stokes Phenomenon and Confluence in Non-autonomous Hamiltonian Systems
}

\author{
Martin Klimeš ${ }^{1}$
}

Received: 29 May 2017 / Accepted: 9 January 2018 / Published online: 20 January 2018

(C) The Author(s) 2018. This article is an open access publication

\begin{abstract}
This article studies a confluence of a pair of regular singular points to an irregular one in a generic family of time-dependent Hamiltonian systems in dimension 2. This is a general setting for the understanding of the degeneration of the sixth Painlevé equation to the fifth one. The main result is a theorem of sectoral normalization of the family to an integrable formal normal form, through which is explained the relation between the local monodromy operators at the two regular singularities and the non-linear Stokes phenomenon at the irregular singularity of the limit system. The problem of analytic classification is also addressed.
\end{abstract}

Keywords Non-autonomous Hamiltonian systems · Irregular singularity · Non-linear Stokes phenomenon - Wild monodromy · Confluence $\cdot$ Local analytic classification . Painlevé equations

Mathematics Subject Classification $34 \mathrm{M} 35 \cdot 34 \mathrm{M} 40 \cdot 34 \mathrm{M} 55$

\section{Introduction}

We consider a parametric family of non-autonomous Hamiltonian systems of the form

$$
\begin{aligned}
& x(x-\epsilon) \frac{d y_{1}}{d x}=\frac{\partial H}{\partial y_{2}}(y, x, \epsilon) \\
& x(x-\epsilon) \frac{d y_{2}}{d x}=-\frac{\partial H}{\partial y_{1}}(y, x, \epsilon),
\end{aligned} \quad(y, x, \epsilon) \in\left(\mathbb{C}^{2} \times \mathbb{C} \times \mathbb{C}, 0\right),
$$

$凶$ Martin Klimeš

klmm@seznam.cz; martin.klimes@univie.ac.at

1 Faculty of Mathematics, University of Vienna, Oskar-Morgenstern-Platz 1, 1090 Vienna, Austria 
shortly written as

$$
x(x-\epsilon) \frac{d y}{d x}=J^{\mathrm{t}}\left(D_{y} H\right), \quad J=\left(\begin{array}{cc}
0 & 1 \\
-1 & 0
\end{array}\right),
$$

with a singular Hamiltonian function $\frac{H(y, x, \epsilon)}{x(x-\epsilon)}$, where $H(y, x, \epsilon)$ is an analytic germ such that $H(y, 0,0)$ has a non-degenerate critical point (Morse point) at $y=0$ :

$$
D_{y} H(0,0,0)=0, \quad \operatorname{det} D_{y}^{2} H(0,0,0) \neq 0 .
$$

The last condition means that the $y$-linear terms of the right side of (2) are of the form $A(x, \epsilon) y$ for $A(x, \epsilon)=J D_{y}^{2} H(0, x, \epsilon)$, with $\operatorname{tr} A(x, \epsilon) \equiv 0$ and $\operatorname{det} A(0,0) \neq 0$, i.e. $A(0,0) \sim\left(\begin{array}{cc}\lambda^{(0)}(0) & 0 \\ 0 & -\lambda^{(0)}(0)\end{array}\right)$ for some $\lambda^{(0)}(0) \neq 0$.

For $\epsilon \neq 0$ the system (1) has two regular singular points at $x=0$ and $x=\epsilon$. At each one of them, the local information about the system is carried by a formal invariant and a monodromy (holonomy) operator. On the other hand, for $\epsilon=0$ the corresponding information about the irregular singularity at $x=0$ is carried by a formal invariant and by a pair of non-linear Stokes operators. Our main goal is to explain the relation between these two distinct phenomena, and to show how the Stokes operators are related to the monodromy operators. The principal thesis is, that while the monodromy operators diverge when $\epsilon \rightarrow 0$, they each accumulate to a 1-parameter family of "wild monodromy operators" which encode the Stokes phenomenon (Theorem 40). It is expected that this "wild monodromy" should have Galoisian interpretation, this question is however outside the reach of this paper.

An important example of a confluent family of systems (1), which in fact motivates this study, is the degeneration of the sixth Painlevé equation to the fifth one, presented in Sect. 6. A more detailed treatment of this confluence will be the subject of an upcoming article [14], which will provide an explicit description of a representation of the "wild monodromy" of the fifth Painlevé equation as an action on the "wild character variety" of the associated isomonodromic problem.

The main tool in our investigation is a theorem on "sectoral normalization" of the family (Theorem 17) to its formal normal form which is integrable. The domains on which these transformations exist are of the form introduced in recent years by C. Rousseau and her collaborators $[10,15,16,25,26]$, consisting of two sectors in the $\epsilon$-space and a family of ramified domains in the $x$-space attached to both singular points $x=0, \epsilon$ along certain logarithmic spirals. When $\epsilon \rightarrow 0$ they tend to a pair of usual sectors attached to the irregular singularity at $x=0$. This construction allows to define unfolded Stokes operators for the family, analogical to those introduced in $[10,15]$ for linear systems, and to decompose the nonlinear monodromy operators around 0 and $\epsilon$ for $\epsilon \neq 0$ into a convergent "Stokes" part and a wild "exponential torus" part (Theorem 38). We also provide an analytic classification for the family (Theorem 36), where the analytic modulus is expressed in terms of a conjugacy class of the unfolded Stokes operators. 
In Sect. 5, we illustrate all this on the example of 2-dimensional traceless linear differential systems

$$
x(x-\epsilon) \frac{d y}{d x}=A(x, \epsilon) y, \quad A(x, \epsilon) \in \mathfrak{s l}_{2}(\mathbb{C}), \quad \operatorname{det} A(0,0) \neq 0,
$$

for which our description follows from the more general work of Lambert and Rousseau $[10,15]$. Here the relation between the monodromy of the unfolded system and the Stokes operators of the limit systems can be summarized as:

Theorem When $\epsilon \rightarrow 0$ the elements of the monodromy group of the system (3) accumulate to generators of the wild monodromy group of the limit system (that is the group generated by the Stokes operators and the exponential torus).

The non-linear analogy of this theorem is Theorem 40. The linear case can be kept in mind as a leading example of which the general non-linear case is a close parallel.

\section{The Foliation and its Formal Invariants}

The family of systems (1) defines a family of singular foliations in the $(y, x)$-space, leaves of which are the solutions. We associate to (1) a family of vector fields tangent to the foliation

$$
Z_{H}(y, x, \epsilon)=x(x-\epsilon) \partial_{x}+X_{H}(y, x, \epsilon)
$$

where

$$
X_{H}(y, x, \epsilon)=\frac{\partial H}{\partial y_{2}}(y, x, \epsilon) \partial_{y_{1}}-\frac{\partial H}{\partial y_{1}}(y, x, \epsilon) \partial_{y_{2}},
$$

which may also be seen as a single vector field in the $(y, x, \epsilon)$-space. We will denote

$$
Z_{H, \epsilon}(y, x), \text { resp. } X_{H, x, \epsilon}(y),
$$

the restriction of (4) to a fixed value of $\epsilon$, resp. of (5) to a fixed value of $(x, \epsilon)$.

The vector field $Z_{H, 0}(y, x)$ has a saddle-node type singularity at $(y, x)=0$, i.e. its linearization matrix has one zero eigenvalue, corresponding to the $x$-direction. It follows from the Implicit Function Theorem that, for small $\epsilon \neq 0, Z_{H, \epsilon}$ has two singular points $\left(y_{0}(0, \epsilon), 0\right)$ and $\left(y_{0}(\epsilon, \epsilon), \epsilon\right)$ bifurcating from $\left(y_{0}(0,0), 0\right)=0$ and depending analytically on $\epsilon$. The aim of this paper is a study of their confluence when $\epsilon \rightarrow 0$.

The two singularities of $Z_{H, \epsilon}$ have each a strong invariant manifold $\mathrm{Y}_{0}=\{(y, x)$ : $x=0\}$, resp. $\mathrm{Y}_{\epsilon}=\{(y, x): x=\epsilon\}$. Away of these invariant manifolds the vector field $Z_{H, \epsilon}$ is transverse to the fibration with fibers $Y_{c}=\{(y, x): x=c\}$. The $(y, x)$-space is endowed with a Poisson structure associated to the 2 -form

$$
\omega=d y_{1} \wedge d y_{2}
$$

the restriction of which on each fiber $Y_{c}$ is symplectic. The vector field $Z_{H, \epsilon}$ is transversely Hamiltonian with respect to this fibration, the form $\omega$, and the Hamiltonian function $H(y, x, \epsilon)$. 


\subsection{Fibered Changes of Coordinates}

We consider the problem of analytic classification of families of systems (1), or orbital analytic classification of vector fields (4), with respect to fiber-preserving (shortly fibered) changes of coordinates

$$
(y, x, \epsilon)=(\Phi(u, x, \epsilon), x, \epsilon) .
$$

Such a change of coordinate transforms a system (2) to a system

$$
\begin{aligned}
x(x-\epsilon) \frac{d u}{d x} & =\left(D_{u} \Phi\right)^{-1} J^{\mathrm{t}}\left(D_{y} H\right) \circ \Phi-x(x-\epsilon)\left(D_{u} \Phi\right)^{-1} \frac{\partial \Phi}{\partial x} \\
& =\left(\operatorname{det} D_{u} \Phi\right)^{-1} J^{\mathrm{t}}\left(D_{u}(H \circ \Phi)\right)-x(x-\epsilon)\left(D_{u} \Phi\right)^{-1} \frac{\partial \Phi}{\partial x},
\end{aligned}
$$

using the identity

$$
P J{ }^{\mathrm{t}} P=\operatorname{det} P \cdot J \text { for any } 2 \times 2 \text { matrix } P \text {. }
$$

Definition 1 We call a fibered transformation $\Phi$ transversely symplectic if $\operatorname{det}\left(D_{u} \Phi\right)$ $\equiv 1$, i.e. if it preserves the restriction of $\omega$ to each fiber $Y_{x}$.

Definition 2 Two systems (1) with Hamiltonian functions $\frac{H(y, x, \epsilon)}{x(x-\epsilon)}$ and $\frac{\tilde{H}(u, x, \epsilon)}{x(x-\epsilon)}$ are called analytically equivalent if there exists an analytic germ of a transversely symplectic transformation $y=\Phi(u, x, \epsilon)$ that is analytic in $(u, x, \epsilon)$ and transforms one system to another: $\Phi^{*} Z_{H, \epsilon}=Z_{\tilde{H}, \epsilon}$.

Lemma 3 If a transformation $y=\Phi(u, x, \epsilon)$ is transversely symplectic, then the transformed system (7) is transversely Hamiltonian w.r.t. $\omega=d u_{1} \wedge d u_{2}$, i.e. it is again of the form (1).

Proof It is enough to show that the system $\frac{d u}{d x}=-\left(D_{u} \Phi\right)^{-1} \frac{\partial \Phi}{\partial x}$ is transversely Hamiltonian, that is, denoting $\left(\begin{array}{l}f_{1} \\ f_{2}\end{array}\right):=-\left(D_{u} \Phi\right)^{-1} \frac{\partial \Phi}{\partial x}$, to show that $\partial u_{1} f_{1}+\partial_{u_{2}} f_{2}=$ 0 . Using the identity (8), we can express $f_{1}=-{\frac{1}{\operatorname{det} D_{u} \Phi}}^{\mathrm{t}}\left(\partial_{u_{2}} \Phi\right) J \partial_{x} \Phi, \quad f_{2}=$ $\frac{1}{\operatorname{det} D_{u} \Phi}{ }^{\mathrm{t}}\left(\partial_{u_{1}} \Phi\right) J \partial_{x} \Phi$, hence

$$
\begin{aligned}
\partial_{u_{1}} f_{1}+\partial_{u_{2}} f_{2} & =\frac{-1}{\operatorname{det} D_{u} \Phi}\left[{ }^{\mathrm{t}}\left(\partial_{u_{2}} \Phi\right) J \partial_{x} \partial_{u_{1}} \Phi-{ }^{\mathrm{t}}\left(\partial_{u_{1}} \Phi\right) J \partial_{x} \partial_{u_{2}} \Phi\right] \\
& =\frac{-1}{\operatorname{det} D_{u} \Phi} \partial_{x}\left[{ }^{\mathrm{t}}\left(\partial_{u_{2}} \Phi\right) J \partial_{u_{1}} \Phi\right]=\frac{-1}{\operatorname{det} D_{u} \Phi} \partial_{x} \operatorname{det} D_{u} \Phi=0 .
\end{aligned}
$$

\subsection{The Formal Invariant $\chi(h, x, \epsilon)$}

Theorem 4 (Siegel) Let $H:\left(\mathbb{C}^{2}, 0\right) \rightarrow(\mathbb{C}, 0)$ have a non-degenerate critical point at 0 , and let $\omega$ be a symplectic volume form. There exists an analytic system of coordinates $u=\left(u_{1}, u_{2}\right)$ in which 


$$
\omega=d u_{1} \wedge d u_{2}, \text { and } H=G_{H}\left(u_{1} u_{2}\right), \quad G_{H}(0)=0 .
$$

The function $G_{H}$ is uniquely determined by the pair $(H, \omega)$ up to the involution

$$
G_{H}\left(u_{1} u_{2}\right) \mapsto G_{H}\left(-u_{1} u_{2}\right),
$$

induced by the symplectic change of variable $J:\left(u_{1}, u_{2}\right) \mapsto\left(u_{2},-u_{1}\right)$. The pair $\left(G_{H}, \omega\right)$ is called the Birkhoff-Siegel normal form of the pair $(H, \omega)$. Moreover, if $(H, \omega)$ depend analytically on a parameter, then so does $G_{H}$ and the change of coordinates.

Proof While not explicitly stated in this form, the existence part of the theorem is originally proved by Siegel [28, chaps. 16 and 17]. See also [8,36]. The uniqueness can be seen by expressing $G_{H}(h)$ in terms of a period map of $\frac{\omega}{d H}$ over a vanishing cycle, see Sect. 2.2.1 below.

Remark 5 - The Theorem 4 provides the existence of an analytic transformation of a Hamiltonian vector field $\dot{y}=J^{\mathrm{t}}\left(D_{y} H\right)$ in dimension 2 to its Birkhoff normal form $\dot{u}=J^{\mathrm{t}}\left(D_{u} G_{H}\right)$, with

$$
G_{H}(h)=\lambda h+\cdots,
$$

where $\pm \lambda \neq 0$ are the eigenvalues of the linear part $J D_{y}^{2} H(0)$. The involution (9) corresponds to the freedom of choice of the eigenvalue $\lambda$.

- The change of coordinates is far from unique. Indeed, the flow of any vector field $\xi=a\left(u_{1} u_{2}\right)\left(u_{1} \partial_{u_{1}}-u_{2} \partial_{u_{2}}\right)$ preserves the normal form.

Let $H=H(y, x, \epsilon)$ be our germ. By the implicit function theorem, for each small $(x, \epsilon)$, the function $H(\cdot, x, \epsilon)$ has an isolated non-degenerate critical point $y_{0}(x, \epsilon)$, depending analytically on $(x, \epsilon)$. Let $y=\Phi(u, x, \epsilon)$ be the transformation to the Birkhoff-Siegel normal form for the function $y \mapsto H(y, x, \epsilon)$ and the form $\omega=d y_{1} \wedge d y_{2}$, depending analytically on $(x, \epsilon)$, i.e.

$$
H(\cdot, x, \epsilon) \circ \Phi(u, x, \epsilon)=G_{H}\left(u_{1} u_{2}, x, \epsilon\right), \quad \operatorname{det} D_{u} \Phi(u, x, \epsilon) \equiv 1 .
$$

By (7), it brings the system (1) to a prenormal form

$$
\begin{aligned}
x(x-\epsilon) \frac{d u}{d x} & =J^{\mathrm{t}}\left(D_{u} G_{H}\right)+O(x(x-\epsilon)) \\
& =\chi\left(u_{1} u_{2}, x, \epsilon\right)\left(\begin{array}{cc}
1 & 0 \\
0 & -1
\end{array}\right) u+O(x(x-\epsilon)),
\end{aligned}
$$

where

$$
\chi(h, x, \epsilon)=\chi^{(0)}(h, \epsilon)+x \chi^{(1)}(h, \epsilon):=\frac{\partial G_{H}}{\partial h}(h, x, \epsilon) \bmod x(x-\epsilon),
$$


$h=u_{1} u_{2}$, or equivalently,

$$
\chi(h, x, \epsilon)=\left\{\begin{array}{l}
\frac{1}{\epsilon}\left[x \frac{\partial G_{H}}{\partial h}(h, \epsilon, \epsilon)-(x-\epsilon) \frac{\partial G_{H}}{\partial h}(h, 0, \epsilon)\right], \quad \epsilon \neq 0, \\
\frac{\partial G_{H}}{\partial h}(h, 0,0)+x \frac{\partial^{2} G_{H}}{\partial h \partial x}(h, 0,0), \quad \epsilon=0 .
\end{array}\right.
$$

Definition 6 The function $\chi(h, x, \epsilon)$ is called the formal invariant of the system (1).

For $\epsilon \neq 0$ the formal invariant $\chi$ is completely determined by the functions $G_{H}(\cdot, 0, \epsilon)$ and $G_{H}(\cdot, \epsilon, \epsilon)$, which are analytic invariants of the autonomous Hamiltonian systems $X_{H, 0, \epsilon}, X_{H, \epsilon, \epsilon}(5)$ on the strong invariant manifolds $\mathrm{Y}_{0}, \mathrm{Y}_{\epsilon}$.

Corollary 7 The formal invariant $\chi(h, x, \epsilon)$ is well-defined up to the involution

$$
J^{*}: \chi(h, x, \epsilon) \mapsto-\chi(-h, x, \epsilon),
$$

induced by the symplectic transformation $u \mapsto J u$. It is uniquely determined by the polar part of the Hamiltonian $\frac{H(y, x, \epsilon)}{x(x-\epsilon)}$, and it is invariant with respect to fibered transversely symplectic changes of coordinates.

Let

$$
\lambda(x, \epsilon)=\chi(0, x, \epsilon) .
$$

Then $\pm \lambda(x, \epsilon)$ are the eigenvalues of the matrix of the linearized system $A(x, \epsilon)=$ $J^{\mathrm{t}} D_{y}^{2} H\left(y_{0}(x, \epsilon), x, \epsilon\right)$ modulo $x(x-\epsilon)$, see Example 8, and the involution (13) corresponds to the freedom of choice of the eigenvalue $\lambda$.

Example 8 (Traceless linear systems) A traceless linear system

$$
x(x-\epsilon) \frac{d y}{d x}=A(x, \epsilon) y,
$$

with $\operatorname{tr} A(x, \epsilon)=0$ and $A(0,0) \sim\left(\begin{array}{cc}\lambda^{(0)}(0) & 0 \\ 0 & -\lambda^{(0)}(0)\end{array}\right)$ for some $\lambda^{(0)}(0) \neq 0$, is of the form (1) for the quadratic form $H(y, x, \epsilon)=\frac{1}{2}{ }^{\mathrm{t}} y J^{\mathrm{t}} A(x, \epsilon) y$. Let $\pm \tilde{\lambda}(x, \epsilon)$ be the eigenvalues of $A(x, \epsilon)$, and let $C(x, \epsilon)$ be a corresponding matrix of eigenvectors of $A(x, \epsilon)$, depending analytically on $(x, \epsilon)$ and normalized so that $\operatorname{det} C(x, \epsilon)=1$. The change of variable $y=C(x, \epsilon) u$ is the one which brings $\left(H, d y_{1} \wedge d y_{2}\right)$ to its Birkhoff-Siegel normal form $\left(G_{H}=\frac{1}{2} \tilde{\lambda}(x, \epsilon) h^{2}, d u_{1} \wedge d u_{2}\right)$. It transforms the system (14) to a prenormal form

$$
x(x-\epsilon) \frac{d u}{d x}=\left[\tilde{\lambda}(x, \epsilon)\left(\begin{array}{cc}
1 & 0 \\
0 & -1
\end{array}\right)+x(x-\epsilon) C^{-1} \frac{d C}{d x}\right] u .
$$

Denoting $\lambda(x, \epsilon):=\left(\lambda^{(0)}(\epsilon)+x \lambda^{(1)}(\epsilon)\right)=\tilde{\lambda}(x, \epsilon) \bmod x(x-\epsilon)$, then we have $\chi(h, x, \epsilon)=\lambda(x, \epsilon)$ up to the choice of sign (13). It coincides with the formal invariant of $[10,15]$. 


\subsubsection{Geometric Interpretation of the Invariant $\chi$.}

For each small $(x, \epsilon)$, the function $H(\cdot, x, \epsilon)$ has an isolated non-degenerate critical point $y_{0}(x, \epsilon)$, depending analytically on $(x, \epsilon)$, with a critical value $h_{0}(x, \epsilon)$. For $(x, \epsilon)$ fixed, $h \in\left(\mathbb{C}, h_{0}\right)$, consider the germ of the level set $S_{h}(x, \epsilon)=\{y \in$ $\left.\left(\mathbb{C}^{2}, y_{0}(x, \epsilon)\right): H(y, x, \epsilon)=h\right\} \subset \mathrm{Y}_{x}$. As a basic fact of the Picard-Lefschetz theory [1], we know that if $h$ is a non-critical value for $H(\cdot, x, \epsilon)$, i.e. $h \neq h_{0}$, then $S_{h}(x, \epsilon)$ has the homotopy type of a circle. Let $\gamma_{h}(x, \epsilon)$ depending continuously on $(x, \epsilon)$ be a loop generating the first homology group of $S_{h}(x, \epsilon)$, the so called vanishing cycle. And let $\mu$ be a 1 -form such that $\omega=d H \wedge \mu$; its restriction to a non-critical level $S_{h}(x, \epsilon)$ is called the Gelfand-Leray form of $\omega$ and is denoted

$$
\mu=\frac{\omega}{d H}
$$

Its period function over the vanishing cycle

$$
p(h, x, \epsilon):=\frac{1}{2 \pi i} \int_{\gamma_{h}(x, \epsilon)} \frac{\omega}{d H},
$$

is well-defined up to a sign change (orientation of $\gamma_{h}$ ), and depends analytically on $(x, \epsilon)$ [1, chap. 10]. Let $G_{H}(\cdot, x, \epsilon)$ be the inverse of the function $h \mapsto$ $\int_{h_{0}(x, \epsilon)}^{h} p(s, x, \epsilon) d s$. Then $\left(G_{H}, \omega\right)$ is the Birkhoff-Siegel normal form of $(H, \omega)$.

Indeed, the above formula for $G_{H}$ is invariant with respect to analytic transversely symplectic changes of coordinates: Supposing that $H=g\left(y_{1} y_{2}, x, \epsilon\right)$ is in its Birkhoff-Siegel normal form, then the level sets are written as $S_{h}=\left\{y_{1} \neq\right.$ $\left.0, \quad y_{2}=\frac{g^{\circ(-1)}(h, x, \epsilon)}{y_{1}}\right\}$, and $\frac{\omega}{d H}=\frac{d y_{1}}{y_{1} \cdot \frac{\partial g}{\partial\left(y_{1} y_{2}\right)} \circ g^{\circ(-1)}(h, x, \epsilon)}$, and therefore $p(h)=$ $\left(\frac{\partial g}{\partial\left(y_{1} y_{2}\right)}\right)^{-1} \circ g^{\circ(-1)}(h, x, \epsilon)$, i.e. $G_{H}=g$.

The above formula for the Birkhoff-Siegel normal form and hence for the formal invariant $\chi$ involves a double inversion which makes it difficult to calculate. The following proposition, which will be proved in Sect. 7.4 , allows to determine it in some special cases. This will be useful in the case of the fifth Painlevé equation (Sect. 6).

Proposition 9 (Birkhoff-Siegel normal form of an autonomous Hamiltonian system) Let $H(y)$ be of the form

$$
H(y)=G(h)+y_{i} \Delta\left(y_{i}, h\right), \quad h=y_{1} y_{2},
$$

for some $i \in\{1,2\}$, with $G, \Delta$ analytic germs, and

$$
G(h)=\lambda h+O\left(h^{2}\right), \quad \lambda \neq 0 .
$$

Then $(G, \omega)$ is the Birkhoff-Siegel normal form for the pair $(H, \omega), \omega=d y_{1} \wedge d y_{2}$. 
Corollary 10 (Invariant $\chi$ for $\epsilon=0$ ) For $\epsilon=0$, suppose that

$$
H(y, x, 0)=G^{(0)}(h, 0)+x H^{(1)}(y, 0)+O\left(x^{2}\right), \quad h=y_{1} y_{2},
$$

where $G^{(0)}(h, 0)=\lambda^{(0)}(0) h+O\left(h^{2}\right), \lambda^{(0)}(0) \neq 0$. Write

$$
H^{(1)}(y, 0)=G^{(1)}(h, 0)+y_{1} \Delta_{1}\left(y_{1}, h, 0\right)+y_{2} \Delta_{2}\left(y_{2}, h, 0\right),
$$

with $\Delta_{i}\left(y_{i}, h, 0\right)=O\left(y_{i}\right)$. Then

$$
\chi(h, x, 0)=\frac{\partial}{\partial h}\left(G^{(0)}(h, 0)+x G^{(1)}(h, 0)\right)
$$

is the formal invariant of the vector field $Z_{H, 0}=x^{2} \partial_{x}+X_{H}$ associated to $H$.

Proof Consider a deformation

$$
\begin{aligned}
H(y, x, \epsilon)= & G^{(0)}(h, 0)+x G^{(1)}(h, 0)+x y_{1} \Delta_{1}\left(y_{1}, h, 0\right) \\
& +(x-\epsilon) y_{2} \Delta_{2}\left(y_{2}, h, 0\right)+O(x(x-\epsilon)),
\end{aligned}
$$

and calculate the Birkhoff-Siegel invariants for $H(y, 0, \epsilon), H(y, \epsilon, \epsilon)$, using Proposition 9.

\subsection{Model System (Formal Normal Form)}

Definition 11 (Model family) Let $\chi(h, x, \epsilon)$ be the formal invariant of the system (1). The model family (formal normal form) for the the system (1) is the family of systems

$$
\begin{aligned}
& x(x-\epsilon) \frac{d u_{1}}{d x}=\chi\left(u_{1} u_{2}, x, \epsilon\right) \cdot u_{1} \\
& x(x-\epsilon) \frac{d u_{2}}{d x}=-\chi\left(u_{1} u_{2}, x, \epsilon\right) \cdot u_{2},
\end{aligned}
$$

which is Hamiltonian with respect to the Hamiltonian function $\frac{G\left(u_{1} u_{2}, x, \epsilon\right)}{x(x-\epsilon)}$,

$$
G(h, x, \epsilon)=\int_{0}^{h} \chi(s, x, \epsilon) d s=G_{H}(h, x, \epsilon) \bmod x(x-\epsilon) .
$$

The formal normal form of the family $Z_{H}$ is the associated family of vector fields

$$
Z_{G}(u, x, \epsilon)=x(x-\epsilon) \partial_{x}+\chi\left(u_{1} u_{2}, x, \epsilon\right)\left(u_{1} \partial_{u_{1}}-u_{2} \partial_{u_{2}}\right) .
$$

The system (16) is integrable with the function $h(u)=u_{1} u_{2}$ being its first integral, $Z_{G} \cdot h=0$. The general solutions of (16) are of the form

$$
\begin{aligned}
& u_{1}(x, \epsilon ; c)=c_{1} E_{\chi}\left(c_{1} c_{2}, x, \epsilon\right), \\
& u_{2}(x, \epsilon ; c)=c_{2} E_{\chi}\left(c_{1} c_{2}, x, \epsilon\right)^{-1}, \quad c=\left(c_{1}, c_{2}\right) \in \mathbb{C}^{2},
\end{aligned}
$$


where $E_{\chi}(h, x, \epsilon)=\exp \int_{\infty}^{x} \frac{\chi(h, x, \epsilon)}{x(x-\epsilon)} d x$, namely

$$
E_{\chi}(h, x, \epsilon)= \begin{cases}x^{-\frac{\chi^{(0)}(h, \epsilon)}{\epsilon}}(x-\epsilon)^{\frac{\chi^{(0)}(h, \epsilon)}{\epsilon}+\chi^{(1)}(h, \epsilon)}, & \text { for } \epsilon \neq 0, \\ e^{-\frac{\chi^{(0)}(h, 0)}{x} \chi^{\chi^{(1)}(h, 0)},} & \text { for } \epsilon=0 .\end{cases}
$$

In particular, $E_{\chi}(h, x, \epsilon)$ is analytic in $\epsilon$ near the origin for $x \neq 0$.

\section{Formal and Sectoral Normalization Theorem}

Throughout the text we will denote

$$
\mathrm{Y}=\left\{|y|<\delta_{y}\right\}, \quad \mathrm{U}=\left\{|u|<\delta_{u}\right\}, \quad \mathrm{X}=\left\{|x|<\delta_{x}\right\}, \quad \mathrm{E}=\left\{|\epsilon|<\delta_{\epsilon}\right\},
$$

for some $\delta_{y}, \delta_{u}, \delta_{x}, \delta_{\epsilon}>0$ sufficiently small, ${ }^{1}$ and consider the systems (1), resp. (16), as defined on $\mathrm{Y} \times \mathrm{X} \times \mathrm{E}$, resp. $\mathrm{U} \times \mathrm{X} \times \mathrm{E}$. We will implicitly suppose that $\delta_{\epsilon}<<\delta_{x}$ so that the singular points $(y, x)=\left(y_{0}(0, \epsilon), 0\right)$ and $\left(y_{0}(\epsilon, \epsilon), \epsilon\right)$ of the original foliation are both well inside $\mathrm{Y} \times \mathrm{X}$ for all $\epsilon \in \mathrm{E}_{ \pm}$.

We will now define "sectoral" domains in the $\epsilon$ - and $x$-space on which the system can be normalized. They are of the same type as those introduced in $[10,13,15,16,25]$.

Definition 12 (Family of spiraling sectoral domains $\mathrm{X}_{ \pm}(\epsilon), \epsilon \in \mathrm{E}_{ \pm}$) Let $\eta>0$ be an arbitrarily small constant, and let $\delta_{x}>>\delta_{\epsilon}>0$ be radii of small discs at 0 in the $x$-and $\epsilon$-space. Let $\lambda(x, \epsilon)=\chi(0, x, \epsilon)$, and let

$$
\mathrm{E}_{ \pm}:=\left\{|\epsilon|<\delta_{\epsilon},\left|\arg \left( \pm \frac{\epsilon}{\lambda(0,0)}\right)\right|<\pi-2 \eta\right\} \cup\{0\},
$$

be two sectors in the $\epsilon$-space. For $\epsilon \in \mathrm{E}_{ \pm}$define a domain

$$
\mathrm{X}_{ \pm}(\epsilon)
$$

in the $x$-space as a simply connected ramified domain spanned by the complete real trajectories of the vector fields

$$
e^{i \theta_{ \pm}} \cdot \frac{x(x-\epsilon)}{\lambda(x, \epsilon)} \partial_{x}
$$

that never leave the disc of radius $\delta_{x}$, where the phase $\theta_{ \pm}$varies continuously in the interval

$$
\left\{\begin{array}{l}
\max \left\{0, \arg \left(\frac{ \pm \epsilon}{\lambda(0,0)}\right)\right\}-\frac{\pi}{2}+\eta<\theta_{ \pm}<\min \left\{0, \arg \left(\frac{ \pm \epsilon}{\lambda(0,0)}\right)\right\}+\frac{\pi}{2}-\eta, \text { for } \epsilon \neq 0, \\
\left|\theta_{ \pm}\right|<\frac{\pi}{2}-\eta, \text { for } \epsilon=0
\end{array}\right.
$$

\footnotetext{
1 The constants $\delta_{u}, \delta_{x}, \delta_{\epsilon}$ will appear in Theorem 17 in purely existential way; they're "small enough".
} 


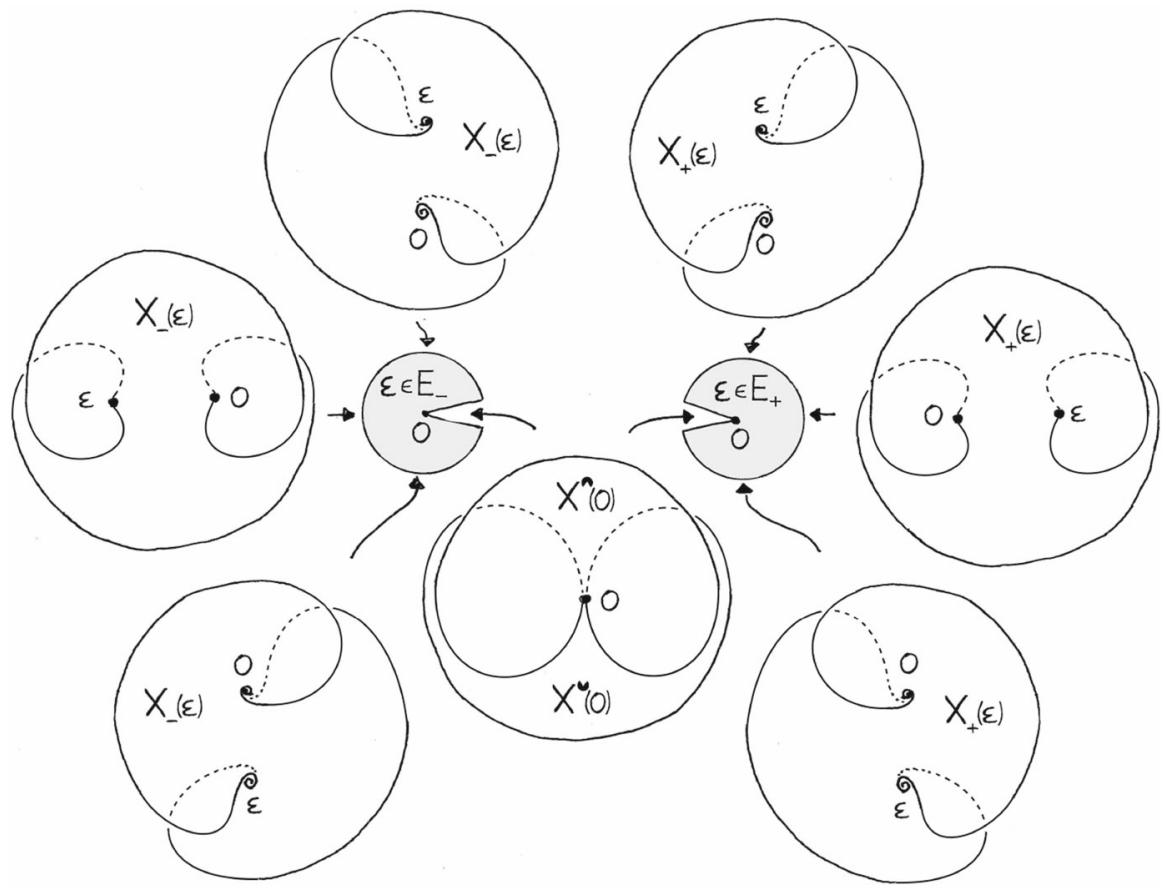

Fig. 1 The domains $X_{ \pm}(\epsilon)$ in dependence on $\epsilon \in E_{ \pm}$. (Picture with $\lambda^{(0)}(0)=1$ )

The constraints (24) on the variation of $\theta_{ \pm}$are such that the real dynamics of the vector field (23) and the asymptotic behavior of the solutions (19) would not change drastically depending on $\theta_{ \pm}$. Namely, for $\epsilon \neq 0$ :

- The point $x=\epsilon$ is repulsive when $\left|\arg \frac{\epsilon}{\lambda(x, \epsilon)}-\theta_{ \pm}\right|<\frac{\pi}{2}$ and attractive when $\left|\arg \frac{-\epsilon}{\lambda(x, \epsilon)}-\theta_{ \pm}\right|<\frac{\pi}{2}$, and vice-versa for the point $x=0$.

- The $u_{1}$-component of the solution (19) tends to 0 along a negative real trajectory of (23) and to $\infty$ along a positive real trajectory for $\left|\theta_{ \pm}\right|<\frac{\pi}{2}$, and vice-versa for the $u_{2}$-component.

For $\epsilon=0$, the domain $\mathrm{X}_{+}(0)=\mathrm{X}_{-}(0)$ consists of a pair of overlapping sectoral domains $^{2} X^{\wedge}(0), X^{\bullet}(0)$ of opening $2 \pi-2 \eta$ with a common point at $x=0$. See Fig. 1. Moreover, it follows from the construction that when $\epsilon \rightarrow 0$ radially with some fixed $\arg \epsilon=\beta$, then $\mathbf{X}_{ \pm}(\epsilon)$ tends to a sectoral subdomain of $\mathbf{X}_{ \pm}(0)$ corresponding to the constraints (24) on the variation of the phase $\theta_{ \pm}$.

Note that by our definition the points $x=0, \epsilon$ are included in $\mathrm{X}_{ \pm}(\epsilon)$.

We denote $\mathrm{XE}_{ \pm}$the union of the domains in the $(x, \epsilon)$-space

$$
\mathrm{XE}_{ \pm}:=\left\{(x, \epsilon) \mid x \in \mathrm{X}_{ \pm}(\epsilon)\right\}
$$

\footnotetext{
2 Our notion of sectoral domains deviates slightly from the usual one by including also the vertex point 0.
} 
Remark 13 Denoting $x_{1, \pm}(\epsilon)$ the attractive equilibrium point of (23) and $x_{2, \pm}(\epsilon)$ the repulsive one,

$$
x_{1,+}(\epsilon)=x_{2,-}(\epsilon)=0, \quad x_{1,-}(\epsilon)=x_{2,+}(\epsilon)=\epsilon,
$$

then

$$
\begin{aligned}
& u_{i}(x, \epsilon) \rightarrow \infty, u_{j}(x, \epsilon) \rightarrow 0,(j=3-i), \text { when } x \rightarrow x_{i, \pm}(\epsilon) \\
& \text { along a real trajectory of }(23) .
\end{aligned}
$$

Remark 14 For each $\epsilon \in \mathrm{E}_{ \pm} \backslash\{0\}, \mathrm{X}_{ \pm}(\epsilon)$ is a simply connected ramified domain. Strictly speaking, the domain $\mathrm{X}_{ \pm}(\epsilon)$ is defined on the Riemann surface of $t(x, \epsilon)=$ $\int_{\infty}^{x} \frac{\lambda(x, \epsilon)}{x(x-\epsilon)} d x$, on which it corresponds to a simply connected domain in the coordinate $t(x, \epsilon)$ obtained as a union of infinite stripes of varying directions $\theta_{ \pm}$for $\epsilon \neq 0$, resp. half-planes with boundaries in directions $\theta_{ \pm}$for $\epsilon=0$. See [13] for more details.

Remark 15 In the variable $x=\epsilon z$, the system (2) takes the form of a singularly perturbed system

$$
\epsilon z(z-1) \frac{d y}{d z}=J^{\mathrm{t}}\left(D_{y} H\right)(y, \epsilon z, \epsilon) .
$$

The domains $z \in \frac{1}{\epsilon} \mathrm{X}_{ \pm}(\epsilon), \epsilon \in \mathrm{E}_{ \pm}$, then roughly correspond to the Stokes domains in the sense of exact WKB analysis [12], where the Stokes curves would be the real separatrices of the point $z=\infty$ of the vector field (23) $e^{i\left(\theta_{ \pm}+\arg \epsilon\right)} \frac{z(z-1)}{\lambda(0,0)} \partial_{z}$ with a fixed phase $\theta_{ \pm}(24)$.

Before giving a general theorem on sectoral normalization for the parametric family (1), let us first state it for the limit system with $\epsilon=0$ which has an irregular singularity of Poincaré rank 1 at $x=0$.

Theorem 16 (Formal and sectoral normalization at $\epsilon=0$ ) The system (1) with $\epsilon=0$ can be brought to its formal normal form (16) through a formal transversely symplectic change of coordinates

$$
(y, x)=(\hat{\Psi}(u, x, 0), x), \quad \hat{\Psi}(u, x, 0)=\sum_{k \geq 0} \psi^{(k, 0)}(u) x^{k},
$$

where $\psi^{(k, 0)}(u)$ are analytic in $u$ on a fixed neighborhood $\cup$ of 0 . This formal series is generally divergent, but it is Borel 1-summable, with a pair of Borel sums $\Psi^{\wedge}(u, x, 0)$ and $\Psi^{\bullet}(u, x, 0)$ defined respectively above the sectors $x \in X^{\wedge}(0), X^{\bullet}(0)$ of Definition 12 (for some $0<\eta<\frac{\pi}{2}$ arbitrarily small and some $\delta_{x}>0$ depending on $\eta$ ), and $u \in \mathrm{U}$. The fibered sectoral transformations $(y, x)=\left(\Psi^{\bullet}(u, x, 0), x\right), \bullet=\bullet, \bullet$, are transversely symplectic and bring the system (1) with $\epsilon=0$ to its formal normal form.

The Theorem 16 is originally due to Takano [32] for systems (1) whose formal invariant is of the form $\chi(h, x)=\lambda^{(0)}+x \chi^{(1)}(h)$. In the case of the irregular singularity of the fifth Painlevé equation it was proved earlier by Takano [30]. Some similar 
and closely related theorems are due to Shimomura [27], Yoshida [34], and recently by Bittmann [3,4], which apply to doubly resonant systems $x^{2} \frac{d y}{d x}=F(y, x)$, with $D_{y} F(0,0)=\lambda^{(0)}(0)\left(\begin{array}{ll}1 & \\ & -1\end{array}\right)$ under the condition that $\Re\left(\operatorname{tr} \frac{\partial}{\partial x} D_{y} F(0,0)\right)>0$. This condition is not satisfied for Hamiltonian systems (1) but nevertheless allows to treat Painlevé equations.

Theorem 17 (Formal and sectoral normalization) Let $Z_{H}(y, x, \epsilon)$ be a family of vector fields (4) and let $\chi(h, x, \epsilon)$ be their formal invariant.

(i) There exists a formal transversely symplectic change of coordinates $(y, x, \epsilon)=$ $(\hat{\Psi}(u, x, \epsilon), x, \epsilon)$ which brings $Z_{H}$ to its formal normal form (18). It is written as a formal power series

$$
\hat{\Psi}(u, x, \epsilon)=\sum_{k, l \geq 0} \psi^{(k, l)}(u) x^{k} \epsilon^{l},
$$

with $\psi^{(k, l)}(u)$ analytic in $u$ on the same fixed neighborhood $U=\left\{\left|u_{1}\right|,\left|u_{2}\right|<\delta_{u}\right\}$ of 0 for some $\delta_{u}>0$, and $\hat{\Psi}(u, 0, \epsilon)=\sum_{l \geq 0} \psi^{(0, l)}(u) \epsilon^{l}$ is convergent on $\mathrm{U} \times \mathrm{E}$ for some $\delta_{\epsilon}>0$. The coefficients $\psi^{(k, l)}$ grow at most factorially in $k+l$ :

$$
\sup _{u \in \bigcup}\left|\psi^{(k, l)}(u)\right| \leq C r^{-(k+l)}(k+l) \text { ! for some } C, r>0 \text {. }
$$

(ii) There exists a transversely symplectic fibered change of coordinates $(y, x, \epsilon)=$ $\left(\Psi_{ \pm}(u, x, \epsilon), x, \epsilon\right)$, with $\Psi_{ \pm}(u, 0, \epsilon)=\hat{\Psi}(u, 0, \epsilon)$, defined for $x$ in the spiraling domain $\mathrm{X}_{ \pm}(\epsilon), \epsilon \in \mathrm{E}_{ \pm}$, of Definition 12 (for some $0<\eta<\frac{\pi}{2}$ arbitrarily small and some $\delta_{x}, \delta_{\epsilon}>0$ depending on $\left.\eta\right)$, and for $u \in U$, which brings $Z_{H}$ to its formal normal form (18). It is uniformly continuous on $\mathrm{U} \times \mathrm{XE}_{ \pm}$(25) and analytic on its interior. When $\epsilon$ tends radially to 0 with $\arg \epsilon=\beta$, then $\Psi_{ \pm}(u, x, \epsilon)$ converges to $\Psi_{ \pm}(u, x, 0)$ uniformly on compact sets of the sub-domain $\lim _{\arg \epsilon=\beta}^{\epsilon \rightarrow 0} \mathrm{X}_{ \pm}(\epsilon) \subseteq \mathrm{X}_{ \pm}(0)$. Note that in our notation $\Psi_{ \pm}(u, x, 0)$ consists of a pair of sectoral transformations $\Psi^{\curvearrowleft}(u, x, 0)$ and $\Psi \bullet(u, x, 0)$; it is a functional cochain using the terminology of $[11,18]$.

(iii) Let $\widetilde{\Psi}(u, x, \epsilon)$ be an analytic extension of the function given by the convergent series

$$
\widetilde{\Psi}(u, x, \epsilon)=\sum_{k, l \geq 0} \frac{\psi^{(k, l)}(u)}{(k+l) !} x^{k} \epsilon^{l}, \quad \psi^{(k, l)} \text { as in (28). }
$$

For each point $(x, \epsilon)$, for which there is $\theta \in]-\frac{\pi}{2}, \frac{\pi}{2}\left[\right.$ such that $\mathbb{B}_{\theta} \cdot(x, \epsilon) \subseteq \mathrm{XE}_{ \pm}$, with $\mathbb{B}_{\theta} \subset \mathbb{C}$ denoting the closed disc with center on $e^{i \theta} \mathbb{R}^{+}$and 0 and 1 on its circumference, we can express $\Psi_{ \pm}(u, x, \epsilon)$ through the following Laplace transform of $\tilde{\Psi}$ :

$$
\Psi_{ \pm}(u, x, \epsilon)=\int_{0}^{+\infty e^{i \theta}} \widetilde{\Psi}(u, s x, s \epsilon) e^{-s} d s .
$$

In particular, $\Psi_{ \pm}(u, x, 0)$ is the pair of sectoral Borel sums $\Psi^{\bullet}(u, x, 0), \Psi^{\bullet}(u, x, 0)$ of the formal series $\hat{\Psi}(u, x, 0)$. 
As a consequence, $\Psi_{ \pm}$and $\hat{\Psi}$ satisfy the same $\left(\partial_{u}, \partial_{x}, \partial_{\epsilon}\right)$-differential relations with meromorphic coefficients.

The proof of the theorem will be given in Sect. 7 .

The transformations $\Psi_{ \pm}$and $\hat{\Psi}$ are unique up to left composition with an analytic symmetry of the model system, see Corollary 32 .

Corollary 18 The system (1) possesses:

(i) A formal first integral given by $h \circ \hat{\Psi}^{\circ(-1)}(y, x, \epsilon)$, where $\hat{\Psi}$ is as above and $h(u)=u_{1} u_{2}$ is a first integral of the model system.

(ii) An actual first integral given by $h \circ \Psi_{ \pm}^{\circ(-1)}(y, x, \epsilon)$ that is bounded and analytic on the domain $\mathrm{XE}_{ \pm}$.

Definition 19 The solution $y=\Psi_{ \pm}(0, x, \epsilon)$ is called ramified center manifold of the vector field $Z_{H}$. It is the unique solution of the corresponding system (1) that is bounded on $\mathrm{X}_{ \pm}(\epsilon)$ (cf. [13]).

Remark 20 More generally, instead of the system (1) one could consider a parametric family of systems unfolding a higher order singularity

$$
\begin{aligned}
& \left(x^{k+1}+\epsilon_{k} x^{k}+\cdots+\epsilon_{0}\right) \frac{d y}{d x}=J^{\mathrm{t}}\left(D_{y} H\right), \\
& J=\left(\begin{array}{cc}
0 & 1 \\
-1 & 0
\end{array}\right), \quad(y, x, \epsilon) \in\left(\mathbb{C}^{2} \times \mathbb{C} \times \mathbb{C}^{k+1}, 0\right),
\end{aligned}
$$

for any $k \geq 1$ under the same non-degeneracy condition on $H$. The definition of the formal invariant $\chi(h, x, \epsilon)=\chi^{(0)}(h, \epsilon)+\cdots+x^{k} \chi^{(k)}(h, \epsilon)$ would then be a straightforward generalization of (11), and it is expected that the same should be also true for the assertions (i) and (ii) of Theorem 17 where the "sectoral" domains in the $(x, \epsilon)$-space should be generalized in the spirit of those introduced in [10].

\section{Stokes Operators and Accumulation of Monodromy}

We will define several operators acting as transversely symplectic fibered isotropies on the three following parametric foliations given by three different vector fields:

- Foliations in the $(u, x)$-space given by the model vector field $Z_{G}(u, x, \epsilon)(18)$.

- Foliations in the $(c, x)$-space, $c$ being the constant of initial condition in (19), given by the rectified vector field $Z_{0}(c, x, \epsilon)=x(x-\epsilon) \partial_{x}$. Note that a fibered isotropy of $Z_{0}$ is necessarily independent of $x$; it acts on the $c$-space of initial conditions only.

- Foliations in the $(y, x)$-space given by the original vector field $Z_{H}(y, x, \epsilon)$ (4).

Notation 21 If $f=f(u, x, \epsilon)$ is a map, we denote $f(\cdot, x, \epsilon): u \mapsto f(u, x, \epsilon)$ the restricted map. Hence $f(\cdot, x, \epsilon) \circ g(\cdot, x, \epsilon)=f(g(\cdot, x, \epsilon), x, \epsilon)$. 


\subsection{Symmetries of the Model System: Exponential Torus}

A vertical infinitesimal symplectic symmetry, or shortly infinitesimal symmetry, of the normal form vector field $Z_{G}$ (18) is an analytic germ of a family of vector fields $\xi(u, x, \epsilon)$ in the $(u, x)$-space that preserves:

(i) the $x$-coordinate: $\mathcal{L}_{\xi} x=\xi \cdot x=0$,

(ii) the symplectic form $\omega=d u_{1} \wedge d u_{2}: \mathcal{L}_{\xi} \omega=0$,

(iii) the vector field $Z_{G}: \quad \mathcal{L}_{\xi} Z_{G}=\left[\xi, Z_{G}\right]=0$.

Similarly, we can define a vertical infinitesimal symplectic symmetry $\xi_{\epsilon}(u, x)$ of the normal form vector field $Z_{G, \epsilon}$ restricted to a fixed $\epsilon$.

Lemma 22 A family of vector fields $\xi(u, x, \epsilon)$ is an infinitesimal symmetry of $Z_{G}$ if and only if there exists an analytic first integral $f(u, x, \epsilon)$ of $Z_{G}$, i.e. $Z_{G} \cdot f=0$, such that $\xi=X_{f}(u, x, \epsilon)$ is a Hamiltonian vector field for $f$ with respect to $\omega$. Similarly for an infinitesimal symmetry $\xi_{\epsilon}$ of $Z_{G, \epsilon}$ for any fixed $\epsilon$.

Proof The conditions (i) and (ii) say that $\xi=a_{1}(u, x, \epsilon) \partial_{u_{1}}+a_{2}(u, x, \epsilon) \partial_{u_{2}}$ with $\frac{\partial a_{1}}{\partial u_{1}}+\frac{\partial a_{2}}{\partial u_{2}}=0$, i.e. $a_{1}=\frac{\partial f}{\partial u_{2}}, a_{2}=-\frac{\partial f}{\partial u_{1}}$ for some $f$ with $f(0, x, \epsilon)=0$, and $\xi=\frac{\partial f}{\partial u_{2}} \partial_{u_{1}}-\frac{\partial f}{\partial u_{1}} \partial_{u_{2}}=X_{f}$. The condition (iii) now says that

$$
0=Z_{G} \cdot D_{u} f-X_{f} \cdot D_{u} G=D_{u}\left(Z_{G} \cdot f\right),
$$

which means that $Z_{G} \cdot f$ is independent of $u$, hence by evaluating at $u=0:\left.Z_{G} \cdot f\right|_{u=0}=$ $\left.x(x-\epsilon) \frac{\partial f}{\partial x}\right|_{u=0}=0$.

The "initial condition" constant $c \in\left(\mathbb{C}^{2}, 0\right)$ in the general solution $u(x, \epsilon ; c)$ (19) is obviously locally constant along the solution, hence it can be seen as a local first integral of the corresponding vector field $Z_{G}$. By expressing it as a function of $(u, x, \epsilon)$, we obtain

$$
c_{1}(u, x, \epsilon)=u_{1} \cdot E_{\chi}(h, x, \epsilon)^{-1}, \quad c_{2}(u, x, \epsilon)=u_{2} \cdot E_{\chi}(h, x, \epsilon),
$$

and

$$
h(u)=u_{1} u_{2}=c_{1} c_{2},
$$

where $E_{\chi}(h, x, \epsilon)$ is as in (20). Clearly, any function of $c=\left(c_{1}, c_{2}\right)$ and $\epsilon$ is again a first integral of $Z_{G}$, and since $c$ defines local coordinates on the space of leaves (space of initial conditions), the converse is also true. Note that the map $c: u \mapsto c(u, x, \epsilon)$ conjugates the vector field $Z_{G}$ to the "rectified" vector field $Z_{0}=x(x-\epsilon) \partial_{x}$ in the $(c, x)$-space:

$$
Z_{G}=c^{*}\left(Z_{0}\right)
$$

It turns out that analytic first integrals are functions of $h=c_{1} c_{2}$ and $\epsilon$ only.

Proposition 23 If $f(u, x, \epsilon)$ is an analytic first integral of $Z_{G}$ on some neighborhood $\mathrm{U} \times \mathrm{X} \times \mathrm{E}$ of 0 , then $f=F\left(u_{1} u_{2}, \epsilon\right)$ with $F$ analytic. Similarly, an analytic first integral $f_{\epsilon}(u, x)$ of $Z_{G, \epsilon}$ on some neighborhood $U \times X$ of 0 is of the form $f_{\epsilon}=F_{\epsilon}\left(u_{1} u_{2}\right)$ for some analytic $F_{\epsilon}$. 
Proof Since $c_{1}(u, x, \epsilon), c_{2}(u, x, \epsilon)$ are local coordinates on the space of leaves, we can write $f(u, x, \epsilon)=g(c(u, x, \epsilon), \epsilon)$ for some analytic germ $g$, which can be decomposed uniquely as $g(c, \epsilon)=g_{0}(h, \epsilon)+c_{1} g_{1}\left(c_{1}, h, \epsilon\right)+c_{2} g_{2}\left(c_{2}, h, \epsilon\right)$ with $g_{l}$ analytic. When $x \rightarrow x_{i, \pm}$ from within $\mathrm{X}_{ \pm}(\epsilon)$, then $c_{i}(u, x, \epsilon) \rightarrow 0$ and $c_{j}(u, x, \epsilon) \rightarrow \infty$ for every $u \in \mathrm{U}$. Since $f(u, x, \epsilon)=g(c(u, x, \epsilon), \epsilon)$ is bounded on $\mathrm{X}_{ \pm}(\epsilon)$, this means that $g_{j}=0$. Therefore $f(u, x, \epsilon)=g_{0}(h, \epsilon)$.

Corollary 24 The Lie algebra tor of analytic infinitesimal symmetries of $Z_{G}$ consists of Hamiltonian vector fields

$$
\mathfrak{t o r}=\left\{\xi=a\left(u_{1} u_{2}, \epsilon\right)\left(u_{1} \partial_{u_{1}}-u_{2} \partial_{u_{2}}\right)=a(h, \epsilon) X_{h}: a(h, \epsilon) \text { analytic }\right\}
$$

and is commutative. It is called the infinitesimal torus of $Z_{G}$.

Similarly, for a fixed $\epsilon \in \mathrm{E}$, the Lie algebra tor $_{\epsilon}$ of analytic infinitesimal symmetries of $Z_{G, \epsilon}$ consists of Hamiltonian vector fields

$$
\mathfrak{t o r}_{\epsilon}=\left\{\xi_{\epsilon}=a_{\epsilon}\left(u_{1} u_{2}\right)\left(u_{1} \partial_{u_{1}}-u_{2} \partial_{u_{2}}\right)=a_{\epsilon}(h) X_{h}: a_{\epsilon}(h) \text { analytic }\right\},
$$

and is commutative. It is called the infinitesimal torus of $Z_{G, \epsilon}$.

The time-1 flow map of a vector field (31) is given by

$$
u \mapsto T_{a}(u):=\Phi_{a X_{h}}^{1}(u)=\left(\begin{array}{cc}
e^{a(h, \epsilon)} & 0 \\
0 & e^{-a(h, \epsilon)}
\end{array}\right) u
$$

Definition 25 We define the exponential torus of $Z_{G}$ as the connected commutative Lie group

$$
\text { Tor }=\exp \text { tor }=\left\{T_{a}: a(h, \epsilon) \text { analytic }\right\},
$$

and likewise, for a fixed $\epsilon \in \mathrm{E}$, the exponential torus of $Z_{G, \epsilon}$ as the commutative Lie group

$$
\operatorname{Tor}_{\epsilon}=\exp \operatorname{tor}_{\epsilon}=\left\{T_{a_{\epsilon}}: a_{\epsilon}(h) \text { analytic }\right\}
$$

Definition 26 A transversely symplectic fibered symmetry, or shortly symmetry, of a family of vector fields $Z_{G}$ is a germ of analytic symplectic transformation $(u, x, \epsilon) \mapsto(\phi(u, x, \epsilon), x, \epsilon)$, such that $\phi^{*} Z_{G}=Z_{G}$. Similarly, for a fixed $\epsilon \in \mathrm{E}$, a symmetry of a vector field $Z_{G, \epsilon}$ is a germ of analytic symplectic transformation $(u, x) \mapsto\left(\phi_{\epsilon}(u, x), x\right)$, such that $\phi_{\epsilon}^{*} Z_{G, \epsilon}=Z_{G, \epsilon}$.

Proposition 27 The (transversely symplectic fibered) symmetries of $Z_{G}$, resp. $Z_{G, \epsilon}$, are exactly the elements of the exponential torus Tor, resp. Tor ${ }_{\epsilon}$.

This follows as an immediate corollary from Proposition 31 below. A characterization of the Lie group of symmetries of a general system (1) will be given in Proposition 34. 
Fig. 2 The intersection sectors $\mathrm{X}_{i, \pm}^{\bigcap}(\epsilon), i=1,2$, for some fixed value of $\epsilon$. The two arrows symbolize the Stokes operators (40) which change the branch of the transformation $\Psi_{ \pm}$above the intersection sectors

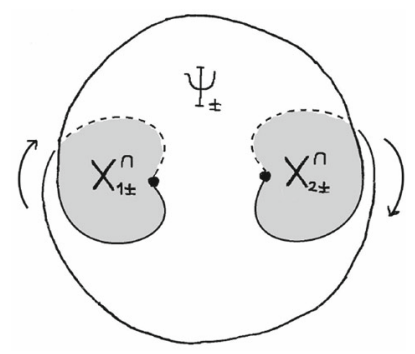

Definition 28 (Intersection sectors) For $\epsilon \in \mathrm{E}_{ \pm} \backslash\{0\}$ define the left and right intersection sectors

$$
\mathbf{X}_{i, \pm}^{\cap}(\epsilon)=\left\{x \in \mathbf{X}_{ \pm}(\epsilon): x_{i, \pm}+e^{2 \pi i}\left(x-x_{i, \pm}\right) \in \mathbf{X}_{ \pm}(\epsilon)\right\}
$$

and for $\epsilon=0$ let $X_{i \pm}^{\cap}(0)$ be their limits (see Fig. 2). They are the domains of self-intersection of $\mathrm{X}_{ \pm}(\epsilon)$ attached to the points $x_{i, \pm}(\epsilon)$ (26). And they are simply connected; in fact in the coordinate $t(x, \epsilon)=\int_{\infty}^{x} \frac{\lambda(x, \epsilon)}{x(x-\epsilon)} d x$ of Remark 14 each intersection sectors corresponds to an "infinite wedge".

Definition 29 An isotropy of a vector field $Z_{G, \epsilon}$ is a germ of transversely symplectic transformation $(u, x) \mapsto\left(\phi_{\epsilon}(u, x), x\right)$, such that $\phi_{\epsilon}^{*} Z_{G, \epsilon}=Z_{G, \epsilon}$. We will consider isotropies of $Z_{G, \epsilon}$ which are analytic and bounded either on the domain $U \times \mathrm{X}_{ \pm}(\epsilon)$ or on $U \times \mathrm{X}_{i, \pm}^{\cap}(\epsilon)$.

Lemma 30 Let $\phi_{\epsilon}(u, x)$ be a sectoral isotropy of the normal form vector field $Z_{G, \epsilon}$, analytic and bounded for $x \in \mathrm{X}_{i, \pm}^{\bigcap}(\epsilon), u \in \mathrm{U}$. Then

$$
c_{i} \circ \phi_{\epsilon}=c_{i} \cdot e^{a_{\epsilon}(h)}+c_{i}^{2} g_{i, \epsilon}\left(h, c_{i}\right), \quad \text { and } c_{j} \circ \phi_{\epsilon}=c_{j} \cdot e^{-a_{\epsilon}(h)}+g_{j, \epsilon}\left(h, c_{i}\right),
$$

for some analytic germs $a_{\epsilon}, g_{i, \epsilon}, g_{j, \epsilon}$, subject to a transversal symplecticity condition $\operatorname{det} D_{c}\left(c \circ \phi_{\epsilon}\right) \equiv 1$.

Proof The isotropy $\phi_{\epsilon}(u, x)$ is analytic in $u$ on some neighborhood of $u=0$ and bounded when $x \rightarrow x_{i, \pm}(\epsilon)$. In particular, the restriction of $c \circ \phi_{\epsilon}$ to any fiber $\{x=$ cst $\neq 0, \epsilon\}$ is analytic in $u$, and therefore the function $c \circ \phi_{\epsilon}(u(x, \epsilon ; c), x)$, which is a function only of $c$, independent of $x$, is analytic in $c$ on some neighborhood of $c=0$.

We have $c_{k}=u_{k} E_{\chi}(h, x, \epsilon)^{(-1)^{k}}, k=1,2,(30)$, and

$$
\lim _{\substack{x \rightarrow x_{i, \pm} \\ x \in \mathbf{X}_{ \pm}(\epsilon)}} E_{\chi}(h, x, \epsilon)^{(-1)^{i}}=0 .
$$

Writing $\phi_{\epsilon}=\left(\phi_{1, \epsilon}, \phi_{2, \epsilon}\right)$, its $i$-th component is given by

$$
\phi_{i, \epsilon}=\left(c_{i} \circ \phi_{\epsilon}\right) \cdot\left(E^{-(-1)^{i}} \circ h \circ \phi_{\epsilon}\right),
$$


and we see that the expansion of $c_{i} \circ \phi_{\epsilon}$ in powers of $c$ can contain only terms $c_{i}^{n_{i}} c_{j}^{n_{j}}=$ $u_{i}^{n_{i}} u_{j}^{n_{j}} E_{\chi}(h, x, \epsilon)^{(-1)^{i}\left(n_{i}-n_{j}\right)}$ with $n_{i} \geq n_{j}+1$, while the expansion of $c_{j} \circ \phi_{\epsilon}$ in powers of $c$ can contain only terms $c_{i}^{n_{i}} c_{j}^{n_{j}}=u_{i}^{n_{i}} u_{j}^{n_{j}} E_{\chi}(h, x, \epsilon)^{(-1)^{i}\left(n_{i}-n_{j}\right)}$ with $n_{i} \geq$ $n_{j}-1$, i.e.

$$
c_{i} \circ \phi_{\epsilon}=c_{i} \cdot e^{a_{i, \epsilon}(h)}+c_{i}^{2} g_{i, \epsilon}\left(h, c_{i}\right), \quad \text { and } \quad c_{j} \circ \phi_{\epsilon}=c_{j} \cdot e^{a_{j, \epsilon}(h)}+g_{j, \epsilon}\left(h, c_{i}\right) .
$$

By transversal symplecticity $1=\operatorname{det} D_{u} \phi_{\epsilon}=\operatorname{det} D_{c}\left(c \circ \phi_{\epsilon}\right)$. One can verify that the last determinant is a function of $\left(h, c_{i}\right)$ only; expressing it for $c_{i}=0$, we have

$$
1=e^{a_{i, \epsilon}+a_{j, \epsilon}}\left(1+h a_{i, \epsilon}^{\prime}+h a_{j, \epsilon}^{\prime}\right)=\frac{d}{d h}\left(h e^{a_{i, \epsilon}+a_{j, \epsilon}}\right),
$$

which implies that $a_{i, \epsilon}+a_{j, \epsilon}=0$.

Note that the hypersurface $\left\{u_{i}=0\right\}=\left\{c_{i}=0\right\}$ consists of all leaves of $Z_{G, \epsilon}$ that are bounded when $x \rightarrow x_{i, \pm}(\epsilon)$ inside $X_{ \pm}(\epsilon)$, and therefore $\phi_{\epsilon}$ must preserve it.

Proposition 31 An isotropy of the normal form vector field $Z_{G, \epsilon}$ that is bounded and analytic on $\mathrm{U} \times \mathrm{X}_{ \pm}(\epsilon)$ is an element of the exponential torus Tor $_{\epsilon}$ (34).

Proof An isotropy $\phi_{\epsilon}(u, x)$ of the model system bounded and analytic on $\mathrm{U} \times \mathrm{X}_{ \pm}(\epsilon)$ is in particular bounded and analytic on $U \times X_{i, \pm}^{\cap}(\epsilon)$, and therefore by Lemma 30, it is such that

$$
c \circ \phi_{\epsilon}={ }^{\mathrm{t}}\left(c_{1} e^{a_{\epsilon}(h)}, c_{2} e^{-a_{\epsilon}(h)}\right), \quad \text { i.e. } \phi_{\epsilon}(u, x)={ }^{\mathrm{t}}\left(u_{1} e^{a_{\epsilon}(h)}, u_{2} e^{-a_{\epsilon}(h)}\right)
$$

for some analytic germ $a_{\epsilon}$.

Corollary 32 The normalizing transformations $\hat{\Psi}$ and $\Psi_{ \pm}$of Theorem 17 are unique modulo right composition with elements of the exponential torus Tor. They are uniquely determined by the analytic germ $\hat{\Psi}(u, 0, \epsilon)=\Psi_{ \pm}(u, 0, \epsilon)$.

Proof If $\Psi_{ \pm}, \Phi_{ \pm}$are two normalizing transformations for $Z_{H}$, then $\phi_{ \pm}(u, \epsilon):=$ $\Psi_{ \pm}^{\circ(-1)} \circ \Phi_{ \pm}(u, x, \epsilon)$ is by Proposition 31 independent of $x$ and such that $\phi_{ \pm}(\cdot, \epsilon) \epsilon$ Tor $_{\epsilon}$. Hence $\phi_{ \pm}(u, \epsilon)=\hat{\Psi}^{\circ(-1)}(\cdot, 0, \epsilon) \circ \hat{\Phi}(u, 0, \epsilon)$ which is analytic in $\epsilon \in \mathrm{E}$ by Theorem 17 (i).

\subsection{Canonical General Solutions}

The model system has a canonical general solution $u(x, \epsilon ; c)(19)$, depending on an "initial condition" parameter $c \in \mathbb{C}^{2}$, uniquely determined by a choice of a branch of the function $E_{\chi}(h, x, \epsilon)(20)$. Correspondingly, $y=\Psi_{ \pm}(u(x, \epsilon ; c), x, \epsilon)$ is a germ of general solution of the original system on $\mathrm{Y} \times \mathrm{X}_{ \pm}(\epsilon)$. In order for this solution to have a continuous limit when $\epsilon \rightarrow 0$, one has to split the domain $X_{ \pm}(\epsilon)$ in two parts, corresponding to the two parts of $\mathrm{X}_{ \pm}(0)$, by making a cut in between the singular 


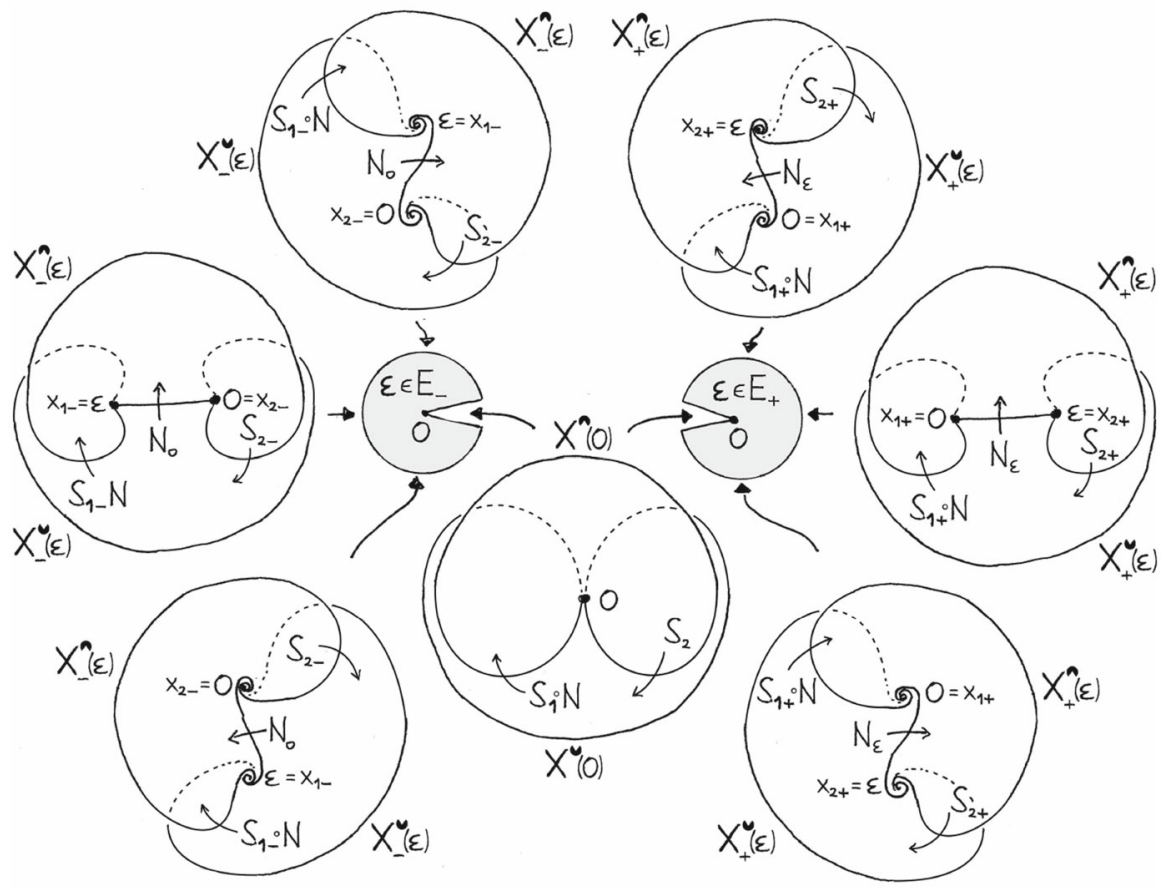

Fig. 3 The sectoral isotropies $S_{1, \pm} \circ N, S_{2, \pm}$ and $N_{x_{2, \pm}}$ as connecting transformations between the canonical general solutions $y_{ \pm}^{\boldsymbol{\wedge}}(x, \epsilon ; \cdot)$ on $\mathbf{X}_{ \pm}^{\wedge}$ and $y_{ \pm}^{\bullet}(x, \epsilon ; \cdot)$ on $\mathbf{X}_{ \pm}^{\boldsymbol{v}}$ above the intersection sectors

points $x_{1, \pm}, x_{2, \pm}$ along a trajectory of (23) through the mid-point $\frac{\epsilon}{2}$ (see Fig. 3). Let us denote $X_{ \pm}^{\wedge}(\epsilon)$ the upper and $X_{ \pm}^{\sim}(\epsilon)$ the lower part (with respect to the oriented line $\left.\lambda^{(0)}(0) \mathbb{R}\right)$ of the cut domain

$$
\mathrm{X}_{ \pm}(\epsilon)=\mathrm{X}_{ \pm}^{\boldsymbol{n}}(\epsilon) \cup \mathrm{X}_{ \pm}^{\boldsymbol{\varpi}}(\epsilon)
$$

The two parts of $\mathrm{X}_{ \pm}(\epsilon)$ intersect in the left and right intersection sectors $\mathrm{X}_{i, \pm}^{\bigcap}(\epsilon)$ (Definition 28) attached to $x_{i, \pm}, i=1,2$, and for $\epsilon \neq 0$ also in a central part along the cut. ${ }^{3}$

Now take two branches $E_{\chi}^{\hat{\wedge}}(h, x, \epsilon)$ and $E_{\chi}^{\bullet}(h, x, \epsilon)$ of $E_{\chi}(h, x, \epsilon)$ on the two parts of the domain, that agree on the right intersection sector $X_{2, \pm}^{\cap}$, and have a limit when $\epsilon \rightarrow 0$. Correspondingly they determine a pair of general solutions of the model system

$$
u^{\bullet}(x, \epsilon ; c), \quad \bullet=\bullet, \bullet
$$

\footnotetext{
3 Technically speaking, the domains $\mathbf{X}_{ \pm}(\epsilon)$ and $\mathbf{X}_{i, \pm}^{\bigcap}(\epsilon)$ are defined on some Riemann surface as explained in Remark 14, what we mean here is the intersection of the projection of $\mathbf{X}_{ \pm}^{\bullet}(\epsilon), \bullet=\boldsymbol{\bullet}, \boldsymbol{\bullet}$, to the $x$-plane.
} 
and a pair of canonical general solutions of the original system

$$
y_{ \pm}^{\bullet}(x, \epsilon ; c):=\Psi_{ \pm}\left(u^{\bullet}(x, \epsilon ; c), x, \epsilon\right), \quad \bullet=\boldsymbol{\bullet}, \boldsymbol{\bullet} .
$$

Since the transformation $\Psi_{ \pm}$is unique only modulo right composition with an exponential torus action $T_{a}(u, \epsilon)(32)$, which acts on $u^{\bullet}(x, \epsilon ; c)$ as

$$
T_{a}(\cdot, \epsilon) \circ u^{\bullet}(x, \epsilon ; c)=u^{\bullet}(x, \epsilon ; \cdot) \circ T_{a}(c, \epsilon),
$$

the solutions $y_{ \pm}^{\bullet}$ are determined only up to the same right action of $T_{a}(c, \epsilon)$.

\subsection{Formal Monodromy}

The formal monodromy operators are induced by monodromy acting on the solutions $u^{\bullet}(x, \epsilon ; c), \bullet=\wedge, \bullet$, of the model system. For $\epsilon \neq 0$ the induced action of formal monodromies along simple counterclockwise loops around each singular point $x_{i, \pm}=$ $0, \epsilon$ on the 3 foliations is given by:

- Monodromy operators of the model system, defined by

$$
\mathscr{N}_{x_{i, \pm}}(\cdot, x, \epsilon) \circ u(x, \epsilon ; c)=u\left(e^{2 \pi i}\left(x-x_{i, \pm}\right)+x_{i, \pm}, \epsilon ; c\right), \quad x \in \mathrm{X}_{ \pm}(\epsilon)
$$

whose action on the foliation of the normal form vector field $Z_{G, \epsilon}$ is given by

$$
\begin{aligned}
& \mathscr{N}_{0}: u \mapsto \exp \left(-2 \pi i \frac{\chi^{0}(h, \epsilon)}{\epsilon}\left(\begin{array}{cc}
1 & 0 \\
0 & -1
\end{array}\right)\right) \cdot u=T_{-\frac{2 \pi i}{\epsilon} \chi \Gamma_{x=0}}(u), \\
& \mathscr{N}_{\epsilon}: u \mapsto \exp \left(2 \pi i\left[\frac{\chi^{0}(h, \epsilon)}{\epsilon}+\chi^{(1)}(h, \epsilon)\right]\left(\begin{array}{cc}
1 & 0 \\
0 & -1
\end{array}\right)\right) \cdot u=T_{\frac{2 \pi i}{\epsilon} \times \Gamma_{x=\epsilon}}(u) .
\end{aligned}
$$

They belong to the exponential torus Tor. The outer monodromy of the model system, that is the monodromy around both $x_{1, \pm}, x_{2, \pm}$, is given by

$$
\mathscr{N}=\mathscr{N}_{0} \circ \mathscr{N}_{\epsilon}=\mathscr{N}_{\epsilon} \circ \mathscr{N}_{0}: u \mapsto \exp \left(2 \pi i \chi^{(1)}\left(u_{1} u_{2}, \epsilon\right)\left(\begin{array}{cc}
1 & 0 \\
0 & -1
\end{array}\right)\right) \cdot u .
$$

- Formal monodromy operators

$$
N_{x_{i, \pm}}(\cdot, \epsilon) \circ c(u, x, \epsilon)=c(\cdot, x, \epsilon) \circ \mathscr{N}_{i, \pm}(u, x, \epsilon),
$$

acting on the space of initial conditions $c$ commutatively by

$$
\begin{aligned}
& N_{0}: c \mapsto \exp \left(-2 \pi i \frac{\chi^{0}(h, \epsilon)}{\epsilon}\left(\begin{array}{cc}
1 & 0 \\
0 & -1
\end{array}\right)\right) \cdot c=T_{-\frac{2 \pi i}{\epsilon} \chi \Gamma_{x=0}}(c), \\
& N_{\epsilon}: c \mapsto \exp \left(2 \pi i\left[\frac{\chi^{0}(h, \epsilon)}{\epsilon}+\chi^{(1)}(h, \epsilon)\right]\left(\begin{array}{cc}
1 & 0 \\
0 & -1
\end{array}\right)\right) \cdot c=T_{\frac{2 \pi i}{\epsilon} \chi \Gamma_{x=\epsilon}}(c),
\end{aligned}
$$


and a formal outer monodromy

$$
N=N_{0} \circ N_{\epsilon}=N_{\epsilon} \circ N_{0}: c \mapsto \exp \left(2 \pi i \chi^{(1)}(h, \epsilon)\left(\begin{array}{cc}
1 & 0 \\
0 & -1
\end{array}\right)\right) \cdot c
$$

- Formal monodromy operators $\mathfrak{N}_{i, \pm}(y, x, \epsilon)$ acting on the foliation of the original vector field $Z_{H, \epsilon}$ :

$$
\mathfrak{N}_{i, \pm}(\cdot, x, \epsilon) \circ \Psi_{ \pm}(u, x, \epsilon)=\Psi_{ \pm}(\cdot, x, \epsilon) \circ \mathscr{N}_{i, \pm}(u, x, \epsilon), \quad x \in \mathrm{X}_{ \pm}(\epsilon)
$$

and

$$
\mathfrak{N}_{ \pm}(\cdot, x, \epsilon)=\mathfrak{N}_{1, \pm}(\cdot, x, \epsilon) \circ \mathfrak{N}_{2, \pm}(\cdot, x, \epsilon)=\mathfrak{N}_{2, \pm}(\cdot, x, \epsilon) \circ \mathfrak{N}_{1, \pm}(\cdot, x, \epsilon)
$$

The canonical solutions $u_{ \pm}^{\wedge}, u_{ \pm}^{\boldsymbol{⿰}}$ of the model system on the domains $\mathrm{X}_{ \pm}^{\boldsymbol{\Lambda}}, \mathrm{X}_{ \pm}^{\boldsymbol{\Lambda}}$, are defined such that they agree on the right intersection sector $X_{2, \pm}^{\cap}$. Therefore on the left intersection sector they are connected by the formal outer monodromy operator

$$
u_{ \pm}^{\curvearrowleft}(x, \epsilon ; c)=\mathscr{N}(\cdot, x, \epsilon) \circ u_{ \pm}^{\swarrow}(x, \epsilon ; c)=u_{ \pm}^{\longleftarrow}(x, \epsilon ; \cdot) \circ N(c, \epsilon), \quad x \in \mathbf{X}_{1, \pm}^{\cap},
$$

and by the formal monodromy $\mathscr{N}_{x_{i, \pm}}$ on the central cut between the two domains for $\epsilon \neq 0$ (cf. Fig. 3).

\subsection{Stokes Operators and Sectoral Isotropies}

Let $y=\Psi_{ \pm}(u, x, \epsilon)$ be the normalizing transformation on $\mathrm{X}_{ \pm}(\epsilon)$. We call Stokes operators the operators that change the determination of the branch of $\Psi_{ \pm}$over the left or right intersection sectors (see Fig. 2). If $x \in \mathbf{X}_{i, \pm}^{\cap}(\epsilon)$, then for $\epsilon \neq 0$ we denote

$$
\bar{x}=e^{2 \pi i}\left(x-x_{i, \pm}\right)+x_{i, \pm}
$$

the corresponding point in $\mathrm{X}_{ \pm}(\epsilon)$ on the other sheet, and extend this notation by limit to $\epsilon=0$. Namely,

$$
\begin{aligned}
& \text { if } x \in \mathrm{X}_{1, \pm}^{\cap}(\epsilon) \subset \mathrm{X}_{ \pm}^{\boldsymbol{\sim}}(\epsilon), \quad \text { then } \bar{x} \in \mathrm{X}_{ \pm}^{\boldsymbol{n}}(\epsilon) \text {, } \\
& \text { if } x \in \mathbf{X}_{2, \pm}^{\cap}(\epsilon) \subset \mathbf{X}_{ \pm}^{\wedge}(\epsilon), \quad \text { then } \bar{x} \in \mathbf{X}_{ \pm}^{\sim}(\epsilon) \text {. }
\end{aligned}
$$

Then the Stokes operators are the operators

$$
\Psi_{ \pm}(u, x, \epsilon) \mapsto \Psi_{ \pm}(u, \bar{x}, \epsilon), \quad x \in \mathrm{X}_{i, \pm}^{\bigcap}(\epsilon),
$$

which for $\epsilon=0$ are the Stokes operators in the usual sense that send the Borel sum of the formal $x$-series $\hat{\Psi}(u, x, 0)$ in one non-singular direction to the Borel sum in a following non-singular direction.

To each of these Stokes operators we associate sectoral isotropies of the 3 foliations. 
- Sectoral isotropies $\mathscr{S}_{i, \pm}(u, x, \epsilon)$ of the normal form vector field $Z_{G, \epsilon}$ :

$$
\Psi_{ \pm}(\cdot, x, \epsilon) \circ \mathscr{S}_{i, \pm}(u, x, \epsilon)=\Psi_{ \pm}(u, \bar{x}, \epsilon), \quad x \in \mathrm{X}_{i, \pm}^{\cap}(\epsilon)
$$

The pair $\left(\mathscr{S}_{1, \pm}, \mathscr{S}_{2, \pm}\right)$ is an analog of the Martinet-Ramis invariant of saddle-node singularity $[17,25]$.

- Sectoral isotropies $S_{1, \pm}(c, \epsilon)$ and $S_{2, \pm}(c, \epsilon)$ of the rectified vector field $Z_{0, \epsilon}=$ $x(x-\epsilon) \partial_{x}$ in the $c$-space:

$$
\begin{array}{ll}
u^{\bullet}(x, \epsilon ; \cdot) \circ S_{1, \pm}(c, \epsilon)=\mathscr{S}_{1, \pm}(\cdot, x, \epsilon) \circ u^{\bullet}(x, \epsilon ; c), & x \in \mathrm{X}_{1, \pm}^{\cap}(\epsilon) \\
u^{\curvearrowleft}(x, \epsilon ; \cdot) \circ S_{2, \pm}(c, \epsilon)=\mathscr{S}_{2, \pm}(\cdot, x, \epsilon) \circ u^{\bullet}(x, \epsilon ; c), & x \in \mathrm{X}_{2, \pm}^{\cap}(\epsilon)
\end{array}
$$

- Sectoral isotropies $\mathfrak{S}_{i, \pm}(y, x, \epsilon)$ of the original vector field $Z_{H, \epsilon}$ :

$$
\mathfrak{S}_{i, \pm}(\cdot, x, \epsilon) \circ \Psi_{ \pm}(u, x, \epsilon)=\Psi_{ \pm}(u, \bar{x}, \epsilon), \quad x \in \mathbf{X}_{i, \pm}^{\cap}(\epsilon)
$$

Proposition 33 (Form of the Stokes isotropies) Let $S_{i, \pm}(c, \epsilon)={ }^{\mathrm{t}}\left(S_{1, i, \pm}(c, \epsilon), S_{2, i, \pm}\right.$ $(c, \epsilon))$ be a Stokes sectoral isotropy (42). Then

- $S_{i, i, \pm}(c, \epsilon)=c_{i}+c_{i}^{2} \cdot \sigma_{i, i, \pm}\left(h, c_{i}, \epsilon\right)$ for an analytic germ $\sigma_{i, i, \pm}$,

- $S_{j, i, \pm}(c, \epsilon)=c_{j}+\sigma_{j, i, \pm}\left(h, c_{i}, \epsilon\right)$ for an analytic germ $\sigma_{j, i, \pm}, j=3-i$,

subject to a condition det $D_{c}\left(S_{i, \pm}\right)=1$.

The term $\sigma_{j, i, \pm}(0,0, \epsilon)$ is responsible for the ramification of the ramified center manifold $y=\Psi_{ \pm}(0, x, \epsilon)$ of the original vector field $Z_{\epsilon}$ at the sector $\mathrm{X}_{i, \pm}^{\cap}(\epsilon)$.

Proof The isotropy $\mathscr{S}_{i, \pm}=\left(\mathscr{S}_{1, i, \pm}, \mathscr{S}_{2, i, \pm}\right)$ is analytic in $u$ on some neighborhood of $u=0$ and bounded in $x$ with $\lim _{x \rightarrow x_{i, \pm}} \mathscr{S}_{k, i, \pm}(u, x, \epsilon)=u_{k}, k=1,2$. By Lemma 30 $S_{k, i, \pm} \circ c=c_{k} \circ \mathscr{S}_{i, \pm}$ is of the form

$$
\begin{aligned}
& c_{i} \circ \mathscr{S}_{i, \pm}=c_{i} \cdot e^{a_{i, \pm}(h, \epsilon)}+c_{i}^{2} \sigma_{i, i, \pm}\left(h, c_{i}, \epsilon\right), \quad \text { and } \\
& c_{j} \circ \mathscr{S}_{i, \pm}=c_{j} \cdot e^{-a_{i, \pm}(h, \epsilon)}+\sigma_{j, i, \pm}\left(h, c_{i}, \epsilon\right)
\end{aligned}
$$

for some some germs $a_{i, \pm}, \sigma_{i, i, \pm}, \sigma_{j, i, \pm}$. We want to show that $a_{i, \pm}=0$.

Writing tautologically

$$
\mathscr{S}_{k, i, \pm}=\left(c_{k} \circ \mathscr{S}_{i, \pm}\right) \cdot\left(E^{-(-1)^{k}} \circ h \circ \mathscr{S}_{i, \pm}\right)
$$

and knowing that $\lim _{x \rightarrow x_{i, \pm}} h \circ \mathscr{S}_{i, \pm}(u, x, \epsilon)=h$, we have $h \circ \mathscr{S}_{i, \pm}=h+c_{i} \cdot(\ldots)$ where $(.$.$) is an analytic function of \left(h, c_{i}, \epsilon\right)$, hence $\lim _{x \rightarrow x_{i, \pm}} E_{\chi}^{-1} \cdot\left(E_{\chi} \circ h \circ \mathscr{S}_{i, \pm}\right)=$ 1, and therefore $\lim _{x \rightarrow x_{i, \pm}}\left(c_{k} \circ \mathscr{S}_{i, \pm}\right) \cdot E^{-(-1)^{k}}=u_{k}$, from which we conclude that $a_{i, \pm}=0$. 


\subsection{Symmetry Group of the System}

The following proposition describes the group of analytic symmetries of the original vector field $Z_{H}$, resp. $Z_{H, \epsilon}$, where the Stokes operators provide an obstruction for analyticity. It is a direct analogue of similar theorems for e.g. saddle-node singularities [25, Proposition 10.6] or for irregular singularities of linear systems [10, Lemma 6.2].

Proposition 34 The group of (analytic transversely symplectic fibered) symmetries of the original vector field $Z_{H}$, resp. its restriction $Z_{H, \epsilon}$ to a fixed $\epsilon$, is either

(1) isomorphic to the exponential torus $\mathbf{T o r}$, resp. $\mathbf{T o r}_{\epsilon}$ : this happens if and only if the system is analytically equivalent to the model (16), or

(2) isomorphic to a finite cyclic group.

If the symmetry group is non-trivial, then the system has an analytic center manifold (bounded analytic solution on a neighborhood of both singular points).

Proof If $\Phi(y, x, \epsilon)$ is a symmetry of the system (1), then $\Phi(\cdot, x, \epsilon) \circ \Psi_{ \pm}(u, x, \epsilon)=$ $\Psi_{ \pm}(\cdot, x, \epsilon) \circ \phi(u, \epsilon)$ for some germ

$$
\phi: u \mapsto\left(\begin{array}{ll}
e^{a(h, \epsilon)} & \\
& e^{-a(h, \epsilon)}
\end{array}\right),
$$

from the exponential torus, and the analyticity of $\Phi$ means that this $\phi$ must commute with the Stokes operators $S_{i, \pm}(42)$ (note that $\phi$ acts the same way on $c$ as on $u$ ). Using their characterization in Proposition 33, this means that

$$
\sigma_{i 1, \pm}\left(h, c_{1}\right)=e^{a} \sigma_{i 1, \pm}\left(h, e^{a} c_{1}\right), \quad \sigma_{i 2, \pm}\left(h, c_{2}\right)=e^{-a} \sigma_{i 2, \pm}\left(h, e^{-a} c_{2}\right), \quad i=1,2 .
$$

This can be satisfied only if

- either $\sigma_{i j, \pm}\left(h, c_{j}\right)=0$ for all $i$, $j$, i.e. if $S_{1, \pm}=\mathrm{id}, S_{2, \pm}=\mathrm{id}$ and the system is analytically equivalent to its formal normal form,

- or there is $k \in \mathbb{N}$ such that $c_{j} \sigma_{i j, \pm}\left(h, c_{j}\right)=0$ contains only powers of $c_{j}^{k}$ for all $i, j$, and $e^{k a}=1$, i.e. $a \in \frac{2 \pi i}{k} \mathbb{Z}$.

\subsection{Analytic Classification}

Definition 35 (Analytic invariants) The collection $\left(\chi,\left\{\mathscr{S}_{1,+}, \mathscr{S}_{2,+}, \mathscr{S}_{1,-}, \mathscr{S}_{2,-}\right\}\right)$ is called an analytic invariant of a system (1). Two analytic invariants $\left(\chi,\left\{\mathscr{S}_{i, \pm}\right\}\right)$, $\left(\tilde{\chi},\left\{\tilde{\mathscr{S}}_{i, \pm}\right\}\right)$ are equivalent if

- either $\chi=\tilde{\chi}$ and there is an element $\phi(u, \epsilon) \in$ Tor of the exponential torus such that: $\mathscr{S}_{i, \pm}=\phi \circ \tilde{\mathscr{S}}_{i, \pm} \circ \phi^{\circ(-1)}, i=1,2$,

- or $\chi(h, x, \epsilon)=-\tilde{\chi}(-h, x, \epsilon)$ and there is an element $\phi(u, \epsilon) \in$ Tor of the exponential torus such that: $\mathscr{S}_{i, \pm}=J \phi \circ \tilde{\mathscr{S}}_{j, \mp} \circ(J \phi)^{\circ(-1)}, i=1,2, j=$ 
$3-i$, where $J:\left(u_{1}, u_{2}\right) \mapsto\left(u_{2},-u_{1}\right)$. Note that the definition of $\mathrm{E}_{ \pm}, \mathrm{X}_{ \pm}$and $x_{i, \pm}$ depends on $\lambda(x, \epsilon)=\chi(0, x, \epsilon)$, therefore the relation $\tilde{\lambda}=-\lambda$ entails the renaming

$$
\tilde{\mathrm{E}}_{ \pm}=\mathrm{E}_{\mp}, \quad \tilde{\mathrm{X}}_{ \pm}^{\bullet}=\mathrm{X}_{\mp}^{\bullet}, \quad \tilde{x}_{i, \pm}=x_{j, \mp} .
$$

By the construction, an analytic invariant of a system (1) is uniquely defined up to the equivalence.

Theorem 36 (Analytic classification) Two systems (1) are analytically equivalent (in the sense of Definition 2) if and only if their analytic invariants are equivalent.

Proof If $y=\Phi(\tilde{y}, x, \epsilon)$ is analytic transformation from one system to another, then the sectoral normalizations $y=\Psi_{ \pm}(u, x, \epsilon)$ and $\tilde{y}=\tilde{\Psi}_{ \pm}(u, x, \epsilon)=\Phi \circ \Psi_{ \pm}$provide the same analytic invariant. Conversely, if the analytic invariants are equivalent, then up to modifying one of the normalizing transformation, one can suppose that they are in fact equal, in which case $\Phi_{ \pm}=\tilde{\Psi}_{ \pm} \circ \Psi_{ \pm}^{\circ(-1)}$ are analytic transformations between the systems on $E_{+}$and $E_{-}$. In fact $\Phi_{+}=\Phi_{-}$is analytic on the whole $\epsilon$-neighborhood E. Indeed, the composition $\Phi_{+} \circ \Phi_{-}^{\circ-1}$ is a symmetry of the second system on the intersection $\mathrm{E}_{+} \cap \mathrm{E}_{-}$, and as such it is determined by its value at $x=0$; but since by Theorem 17 (i) $\tilde{\Psi}_{+}(u, 0, \epsilon)=\tilde{\Psi}_{-}(u, 0, \epsilon)$ and $\Psi_{+}(u, 0, \epsilon)=\Psi_{-}(u, 0, \epsilon)$ are analytic in $\epsilon(29)$, this means that $\Phi_{+} \circ \Phi_{-}^{\circ-1}=$ id.

Example 37 In the case of linear systems (14), the Stokes operators $S_{i, \pm}$ are given by two unipotent matrices

$$
S_{1, \pm}: c \mapsto\left(\begin{array}{cc}
1 & 0 \\
s_{1, \pm}(\epsilon) & 1
\end{array}\right) c, \quad S_{2, \pm}: c \mapsto\left(\begin{array}{cc}
1 & s_{2, \pm}(\epsilon) \\
0 & 1
\end{array}\right) c
$$

In this particular case, the conjugacy class of the outer monodromy $M=S_{2, \pm} S_{1, \pm} N$ is given by $\operatorname{det} M=1, \operatorname{tr} M=2 \cos \left(2 \pi \lambda^{(1)}\right)+e^{2 \pi i \lambda(1)} s_{1, \pm} s_{2, \pm}$, and therefore it determines completely, together with the formal invariant $\lambda(x, \epsilon)$, the analytic invariant of the system.

\subsection{Decomposition of Monodromy Operators}

For $\epsilon \neq 0$, let $x_{0} \in \mathrm{X}_{ \pm}(\epsilon) \backslash\{0, \epsilon\}$ be a base-point, and let two counterclockwise simple loops around the singular points $x_{i, \pm}, i=1,2$, be as in Fig. 4. Correspondingly, we have two monodromy operators $\mathfrak{M}_{x_{i, \pm}}$ acting on the foliation by the solutions of the original system (1) by analytic continuation along the loops. Since the monodromy operators $\mathfrak{M}_{x_{i, \pm}}$ act on the foliation, they are independent of the choice of the twoparameter general solution on which they act on the left (a different general solution is related to it by a change of $c$ independent of $x$, i.e. by right action on the general solution). In particular

$$
\mathfrak{M}_{x_{1,+}}=\mathfrak{M}_{x_{2,-}}, \quad \mathfrak{M}_{x_{2,+}}=\mathfrak{M}_{x_{1,-}}
$$


Fig. 4 Simple loops along which the monodromy operators $\mathfrak{M}_{0}$ and $\mathfrak{M}_{\epsilon}$ are defined

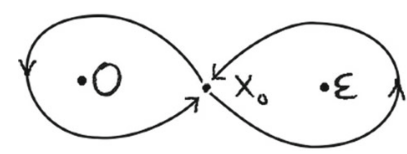

Theorem 38 For $\epsilon \neq 0$, the monodromy operators $\mathfrak{M}_{x_{i, \pm}}$ of the original foliation are well defined on some open neighborhood of the ramified center manifold $y=$ $\Psi_{ \pm}(0, x, \epsilon)$ in $\mathrm{Y} \times \mathrm{X}_{ \pm}(\epsilon)$. Their (left) action is given by

$$
\mathfrak{M}_{x_{i, \pm}}=\mathfrak{S}_{i, \pm} \circ \mathfrak{N}_{i, \pm}
$$

where $\mathfrak{S}_{i, \pm}$ are the Stokes operators $(43)$ and $\mathfrak{N}_{i, \pm}$ are the formal monodromy operators (39). Hence

$$
\begin{aligned}
& \mathfrak{M}_{0}=\mathfrak{S}_{1,+} \circ \mathfrak{N}_{1,+}=\mathfrak{S}_{2,-} \circ \mathfrak{N}_{2,-}, \\
& \mathfrak{M}_{\epsilon}=\mathfrak{S}_{2,+} \circ \mathfrak{N}_{2,+}=\mathfrak{S}_{1,-} \circ \mathfrak{N}_{1,-} .
\end{aligned}
$$

Their right action on analytic extension of the canonical general solutions $y_{ \pm}^{\bullet}$ (35) to the whole $\mathrm{X}_{ \pm}(\epsilon)$ is given by

$$
\mathfrak{M}_{x_{i, \pm}}\left(y_{ \pm}^{\bullet}(x, \epsilon ; c), x, \epsilon\right)=y_{ \pm}^{\bullet}(x, \epsilon ; \cdot) \circ M_{i, \pm}^{\bullet}(c, \epsilon), \quad \bullet=\bullet, \bullet,
$$

where

$$
\begin{array}{ll}
M_{1, \pm}^{\boldsymbol{n}}=N_{x_{2, \pm}}^{\circ(-1)} \circ S_{1, \pm} \circ N, & M_{1, \pm}^{\sim}=S_{1, \pm} \circ N_{x_{1, \pm}}, \\
M_{2, \pm}^{\boldsymbol{n}}=S_{2, \pm} \circ N_{x_{2, \pm}}, & M_{2, \pm}^{\bullet}=N_{x_{2, \pm}} \circ S_{2, \pm},
\end{array}
$$

cf. Fig. 3.

Proof of Theorem 38 The demonstration of the given formulas is straightforward, but we include it here for the sake of completeness. Let $\bar{x}:=x_{i, \pm}+e^{2 \pi i}\left(x-x_{i, \pm}\right)$, then

$$
\begin{aligned}
\mathfrak{M}_{x_{i, \pm}}(\cdot, x, \epsilon) \circ y_{ \pm}(x, \epsilon ; c) & =y_{ \pm}(\bar{x}, \epsilon ; c)=\Psi_{ \pm}(\cdot, \bar{x}, \epsilon) \circ u(\bar{x}, \epsilon ; c) \\
& =\left(\mathfrak{S}_{i, \pm} \circ \Psi_{ \pm}\right)(\cdot, x, \epsilon) \circ u(\bar{x}, \epsilon ; c) \\
& =\left(\mathfrak{S}_{i, \pm} \circ \Psi_{ \pm} \circ \mathscr{N}_{i, \pm}\right)(\cdot, x, \epsilon) \circ u(x, \epsilon ; c) \\
& =\left(\mathfrak{S}_{i, \pm} \circ \mathfrak{N}_{i, \pm} \circ \Psi_{ \pm}\right)(\cdot, x, \epsilon) \circ u(x, \epsilon ; c) \\
& =\left(\mathfrak{S}_{i, \pm} \circ \mathfrak{N}_{i, \pm} \circ y_{ \pm}\right)(x, \epsilon ; c),
\end{aligned}
$$

using (36), (39), (43). Similarly, to calculate $M_{1, \pm}^{\curvearrowleft}$ for example,

$$
\begin{aligned}
y_{ \pm}^{\curvearrowleft}(\bar{x}, \epsilon ; c) & =\Psi_{ \pm}(\cdot, \bar{x}, \epsilon) \circ u^{\curvearrowleft}(\bar{x}, \epsilon ; c) \\
& =\Psi_{ \pm}(\cdot, \bar{x}, \epsilon) \circ u^{\bullet}(\bar{x}, \epsilon ; \cdot) \circ N_{x_{2, \pm}}(c, \epsilon) \\
& =\Psi_{ \pm}(\cdot, \bar{x}, \epsilon) \circ u^{\bullet}(x, \epsilon ; \cdot) \circ N_{x_{1, \pm}} \circ N_{x_{2, \pm}}(c, \epsilon)
\end{aligned}
$$




$$
\begin{aligned}
& =\Psi_{ \pm}(\cdot, x, \epsilon) \circ u^{\bullet}(x, \epsilon ; \cdot) \circ S_{1, \pm} \circ N_{x_{1, \pm}} \circ N_{x_{2, \pm}}(c, \epsilon) \\
& =\Psi_{ \pm}(\cdot, x, \epsilon) \circ u^{\curvearrowleft}(x, \epsilon ; \cdot) \circ N_{x_{2, \pm}}^{\circ(-1)} \circ S_{1, \pm} \circ N_{x_{1, \pm}} \circ N_{x_{2, \pm}}(c, \epsilon) \\
& =y_{ \pm}^{\curvearrowleft}(x, \epsilon ; \cdot) \circ N_{x_{2, \pm}}^{\circ(-1)} \circ S_{1, \pm} \circ N(c, \epsilon),
\end{aligned}
$$

see Fig. 3. The general solutions $y_{ \pm}^{\wedge}, y_{ \pm}^{\bullet}$ are related by

$$
\hat{y_{ \pm}}(x, \epsilon ; c)=y_{ \pm}^{\bullet}(x, \epsilon ; \cdot) \circ N_{x_{2, \pm}}(c, \epsilon) .
$$

Note that in general, a composition of the two monodromies may not be defined if the image of the first does not intersect the domain of definition the second. This is due to the ramification of the center manifold.

\subsection{Accumulation of Monodromy}

\section{Definition 39 (Monodromy pseudogroup)}

(1) For $\epsilon \neq 0$, the pseudogroup generated by the monodromy operators

$$
\left\langle\mathfrak{M}_{0}(\cdot, x, \epsilon), \mathfrak{M}_{\epsilon}(\cdot, x, \epsilon)\right\rangle
$$

is called the (local) monodromy pseudogroup. The pseudogroup generated by the corresponding action on the initial condition $c$

$$
\left\langle M_{1, \pm}^{\bullet}(\cdot, \epsilon), M_{2, \pm}^{\bullet}(\cdot, \epsilon)\right\rangle
$$

is its representation with respect to the general solution $y_{ \pm}^{\bullet}(x, \epsilon ; c)$.

(2) For $\epsilon=0$, the pseudogroup generated by the Stokes operators and by the elements of the exponential torus (pushed-forward by the sectoral transformations $\Psi^{\bullet}$ ):

$$
\left\langle\mathfrak{S}_{1, \pm}(\cdot, x, 0), \mathfrak{S}_{2, \pm}(\cdot, x, 0),\left\{\mathfrak{T}_{a}^{\bullet}(\cdot, x, 0)\right\}_{a}\right\rangle
$$

where $\mathfrak{T}_{a}^{\bullet}(\cdot, x, 0)=\Psi_{ \pm}^{\bullet}(\cdot, x, 0)_{*} T_{a}=\Phi_{\left(\Psi_{ \pm}^{\bullet}\right)_{*}\left(a(h) X_{h}\right)}^{1}$, is called the (local) wild monodromy pseudogroup. The pseudogroup generated by the corresponding action on the initial condition $c$

$$
\left\langle S_{1, \pm}(\cdot, 0), S_{2, \pm}(\cdot, 0),\left\{T_{a}(\cdot)\right\}_{a}\right\rangle,
$$

is its representation with respect to the formal transseries solution $\hat{y}(x, 0 ; c)=$ $\hat{\Psi}(u(x, 0 ; c), x, 0)$.

Note that the pseudogroup (46) is independent of the freedom of choice of the sectoral normalizations $\Psi_{ \pm}^{\bullet}$ of Theorem 16 . 
One of the main goals of this paper is to understand the relation between the monodromy pseudogroup for $\epsilon \neq 0$ and the wild monodromy pseudogroup for $\epsilon=0$.

Suppose that the formal invariant $\chi(h, x, \epsilon)$ is such that

$$
\chi(h, x, 0)=\lambda^{(0)}(0)+x \chi^{(1)}(h, 0),
$$

and therefore

$$
\chi(h, x, \epsilon)=\lambda^{(0)}(0)+\epsilon \frac{\partial \chi^{(0)}}{\partial \epsilon}(h, 0)+x \chi^{(1)}(h, 0)+O(x \epsilon)+O\left(\epsilon^{2}\right) .
$$

Let $\left\{\epsilon_{n}\right\}_{n \in \pm \mathbb{N}}$ be sequence in $\mathrm{E}_{ \pm} \backslash\{0\}$ defined by

$$
\frac{\lambda^{(0)}(0)}{\epsilon_{n}}=\frac{\lambda^{(0)}(0)}{\epsilon_{0}}+n, \quad \epsilon_{0} \in \mathrm{E}_{ \pm} \backslash\{0\}
$$

along which the exponential factor $e^{\frac{2 \pi i \lambda(0)}{\epsilon}}$ in the formal monodromy (38) stays constant, and denote

$$
\kappa:=e^{\frac{2 \pi i \lambda(0)}{\epsilon_{0}}} .
$$

Then the formal monodromy operators $\mathscr{N}_{0}(u, x, \epsilon), \mathscr{N}_{\epsilon}(u, x, \epsilon)$, resp. $N_{0}(u, x, \epsilon), N_{\epsilon}$ $(u, x, \epsilon)$, converge along each such sequence to a symmetry of the model system (element of the exponential torus)

$$
\begin{aligned}
& \tilde{N}_{0}(\kappa ; u, x):=\lim _{n \rightarrow \pm \infty} N_{0}\left(u, x, \epsilon_{n}\right): c \mapsto\left(\begin{array}{cc}
\kappa^{-1} e^{-2 \pi i \frac{\partial \chi^{(0)}}{\partial \epsilon}(h, 0)} & 0 \\
0 & \kappa e^{2 \pi i \frac{\partial \chi^{(0)}}{\partial \epsilon}(h, 0)}
\end{array}\right) c, \\
& \tilde{N}_{\epsilon}(\kappa ; u, x) \\
& \quad:=\lim _{n \rightarrow \pm \infty} N_{\epsilon}\left(u, x, \epsilon_{n}\right): c \mapsto\left(\begin{array}{cc}
\kappa e^{2 \pi i\left[\frac{\partial \chi^{(0)}}{\partial \epsilon}(h, 0)+\chi^{(1)}(h, 0)\right]} & 0 \\
0 & \kappa^{-1} e^{-2 \pi i\left[\frac{\partial \chi^{(0)}}{\partial \epsilon}(h, 0)+\chi^{(1)}(h, 0)\right]}
\end{array}\right) c,
\end{aligned}
$$

$\kappa \in \mathbb{C}^{*}$. This implies that also the monodromy operators $M_{i, \pm}(c, \epsilon)$, resp. $\mathfrak{M}_{x_{i, \pm}}(y, x, \epsilon)$, converge along such sequences $\left\{\epsilon_{n}\right\}_{n \in \pm \mathbb{N}} \subset \mathrm{E}_{ \pm} \backslash\{0\}$. Denote

$$
\tilde{\mathfrak{N}}_{i, \pm}^{\bullet}(\kappa ; y, x):=\lim _{n \rightarrow \pm \infty} \mathfrak{N}_{i, \pm}\left(y, x, \epsilon_{n}\right), \quad x \in \mathbf{X}_{ \pm}^{\bullet}, \quad \bullet=\boldsymbol{\bullet}, \boldsymbol{\bullet}
$$

Theorem 40 Suppose that the formal invariant of the form (47). Then the monodromy operators of the system (1) for $\epsilon \neq 0$ accumulate along the sequences $\left\{\epsilon_{n}\right\}_{n \in \pm \mathbb{N}}$ (48) to a 1-parameter family of wild monodromy operators 


$$
\begin{aligned}
\tilde{\mathfrak{M}}_{1, \pm}(\kappa ; y, x) & :=\lim _{n \rightarrow \pm \infty} \mathfrak{M}_{x_{1, \pm}}\left(y, x, \epsilon_{n}\right) \\
& =\mathfrak{S}_{1, \pm}(\cdot, x, 0) \circ \tilde{\mathfrak{N}}_{x_{1, \pm}}^{\bullet}(\kappa ; y, x), \quad x \in \mathrm{X}_{1, \pm}^{\cap}(0), \\
\tilde{\mathfrak{M}}_{2, \pm}(\kappa ; y, x) & :=\lim _{n \rightarrow \pm \infty} \mathfrak{M}_{x_{2, \pm}}\left(y, x, \epsilon_{n}\right) \\
& =\mathfrak{S}_{2, \pm}(\cdot, x, 0) \circ \tilde{\mathfrak{N}}_{x_{2, \pm}}^{n}(\kappa ; y, x), \quad x \in \mathrm{X}_{2, \pm}^{\cap}(0) .
\end{aligned}
$$

In particular, if we replace $\kappa$ by $e^{-2 \pi i\left[\frac{\partial \chi^{(0)}}{\partial \epsilon}(h, 0)+\delta_{i, \pm}(0) \chi^{(1)}(h, 0)\right]}, \delta_{i, \pm}(\epsilon)=\frac{x_{i, \pm}(\epsilon)}{\epsilon}$, so that $\tilde{\mathfrak{N}}_{x_{i, \pm}}^{\bullet}(\kappa ; y, x)$ becomes an identity, we obtain the Stokes operators

$$
\mathfrak{S}_{i, \pm}(y, x, 0)=\tilde{\mathfrak{M}}_{i, \pm}\left(e^{-2 \pi i\left[\frac{\partial \chi^{(0)}}{\partial \epsilon}(h, 0)+\delta_{i, \pm}(0) \chi^{(1)}(h, 0)\right]} ; y, x\right)
$$

The vector field corresponding to the differential system

$$
\dot{y}= \pm(-1)^{i}\left(\kappa \frac{\partial}{\partial \kappa} \tilde{\mathfrak{M}}_{i, \pm}(\kappa ; y, x)^{\circ(-1)}\right) \circ \tilde{\mathfrak{M}}_{i, \pm}(\kappa ; y, x)
$$

equals to the push-forward $\Psi_{ \pm}^{\bullet}(\cdot, x, 0)_{*}\left(X_{h}\right)$ of the vector field $X_{h}=u_{1} \partial_{u_{1}}-u_{2} \partial_{u_{2}}$, where $\bullet=\backsim$ if $i=1$ and $\bullet=\wedge$ if $i=2$, which "generates" the commutative Lie algebra of bounded infinitesimal symmetries on the sector $\mathrm{X}^{\bullet}(0)$.

Conclusion. The knowledge of the limits $\tilde{\mathfrak{M}}_{i, \pm}(\kappa ; y, x), \kappa \in \mathbb{C}^{*}$, allows to recover the infinitesimal symmetry $\Psi_{ \pm}^{\bullet}(\cdot, x, 0)_{*}\left(X_{h}\right)(52)$, and hence its Hamiltonian, the bounded first integral $\Psi_{ \pm}^{\bullet}(\cdot, x, 0)_{*}(h)$ which vanishes at the singular points $\left(y_{0}\left(x_{i, \pm}, \epsilon\right), x_{i, \pm}\right)$, and therefore, knowing the formal invariant $\chi$, also the formal monodromy operators $\tilde{\mathfrak{N}}_{x_{1, \pm}}^{\bullet}(\kappa ; y, x)$, and finally the Stokes isotropies $\mathfrak{S}_{i, \pm}(y, x, 0)$.

Remark 41 In the case when the assumption (47) is not met, one can nevertheless get a similar "accumulation" result by replacing in $(48) \lambda^{(0)}(0)$ by $\chi^{(0)}(h, 0)$ and defining $\kappa(h)=e^{\frac{2 \pi i \chi^{(0)}(h, 0)}{\epsilon_{0}(h)}}$ (49). In fact, one can interpret the limit $\tilde{\mathfrak{M}}_{i, \pm}$ of $\mathfrak{M}_{x_{i}, \pm}$ along the special sequence $\left\{\epsilon_{n}(h)\right\}_{n \in \pm \mathbb{N}}$ as being obtained by replacing the divergent term $e^{\frac{2 \pi i \chi^{(0)}(h, 0)}{\epsilon}}$ in an expression of $\mathfrak{M}_{x_{i}, \pm}$ by a new function $\kappa(h)$ and taking the limit $\epsilon \rightarrow 0$ of all the other terms bounded in $\epsilon$.

\section{Confluence in $2 \times 2$ Traceless Linear Systems and Their Differential Galois Group}

To illustrate the matter of the previous section, let us consider a confluence of two regular singular points to a non-resonant irregular singular point in a family of linear systems

$$
x(x-\epsilon) \frac{d y}{d x}=A(x, \epsilon) y, \quad y \in \mathbb{C}^{2},
$$


where $A$ is a $2 \times 2$ traceless complex matrix depending analytically on $(x, \epsilon) \in(\mathbb{C} \times$ $\mathbb{C}, 0)$, such that $A(0,0) \neq 0$ has two distinct eigenvalues $\pm \lambda^{(0)}(0)$.

The Theorem 17 in this case can be found in the thesis of Parise [22] and in the work of Lambert and Rousseau [15] (see also [10]). It provides us with a canonical fundamental solution matrices

$$
Y_{ \pm}^{\bullet}(x, \epsilon)=\Psi_{ \pm}(x, \epsilon) \cdot U_{ \pm}^{\bullet}(x, \epsilon), \bullet=\bullet, \bullet,
$$

where the transformation matrix $\Psi_{ \pm}(x, \epsilon)$ is bounded on $\mathrm{XE}_{ \pm}$, and

$$
U_{ \pm}^{\bullet}(x, \epsilon)=\left(\begin{array}{cc}
E_{\lambda}^{\bullet}(x, \epsilon) & 0 \\
0 & E_{\lambda}^{\bullet}(x, \epsilon)^{-1}
\end{array}\right),
$$

is a solution to the diagonal model system

$$
x(x-\epsilon) \frac{d y}{d x}=\left(\begin{array}{ll}
\lambda(x, \epsilon) & -\lambda(x, \epsilon)
\end{array}\right) y .
$$

The solution basis $Y_{ \pm}^{\bullet}(x, \epsilon)$ is also called a mixed basis: the first (resp. second) column spans the subspace of solutions that asymptotically vanish when $x \rightarrow x_{1, \pm}(\epsilon)$ (resp. when $x \rightarrow x_{2, \pm}(\epsilon)$ ), and it is an eigensolution with respect to the corresponding monodromy operator $\mathfrak{M}_{x_{1, \pm}}$ (resp. $\mathfrak{M}_{x_{2, \pm}}$ ) associated to its eigenvalue $e^{ \pm 2 \pi i \frac{\lambda\left(x_{1, \pm}, \epsilon\right)}{\epsilon}}$ (resp. $e^{ \pm 2 \pi i \frac{\lambda\left(x_{2, \pm}, \epsilon\right)}{\epsilon}}$ ). A general solution is a linear combination

$$
y_{ \pm}^{\bullet}(x, \epsilon ; c)=Y_{ \pm}^{\bullet}(x, \epsilon) \cdot c, \bullet=\bullet, \bullet .
$$

Let $\mathbb{K}$ be the field of meromorphic functions of the variable $x$ on a fixed small neighborhood of 0 , equipped with the differentiation $\frac{d}{d x}$. For a fixed small $\epsilon$, the local differential Galois group (also called the Picard-Vessiot group) of the system (53) is the group of $\left(\mathbb{K}, \frac{d}{d x}\right)$-automorphisms of the differential field $\mathbb{K}\langle Y(\cdot, \epsilon)\rangle$, generated by the components of any fundamental matrix solution $Y(x, \epsilon)$. The differential Galois group acts on the foliation associated to the system by left multiplication of solutions of the system. Fixing a fundamental solution matrix $Y=Y_{ \pm}^{\bullet}$, then each automorphism is represented by a right multiplication of $Y_{ \pm}^{\bullet}$ by a constant invertible matrix, hence the differential Galois group is represented by an (algebraic) subgroup of $\mathrm{SL}_{2}(\mathbb{C})$ acting on the right.

It is well known $[19,29]$ that the differential Galois group is the Zariski closure of $\epsilon \neq 0$ : the monodromy group generated by the two monodromy operators around the singular points 0 and $\epsilon$, 
$\epsilon=0$ : the wild monodromy group ${ }^{4}$ generated by the Stokes operators and the linear exponential torus ${ }^{5}$ which acts on the fundamental solutions $Y_{ \pm}^{\bullet}$ as

$$
\mathfrak{T}_{a}^{\bullet}: Y_{ \pm}^{\bullet}(x, 0) \mapsto Y_{ \pm}^{\bullet}(x, 0) \cdot T_{a}, \quad \text { where } T_{a}=\left(\begin{array}{c}
e^{a} \\
e^{-a}
\end{array}\right), \quad a \in \mathbb{C}
$$

The question is how are these two different descriptions related?

The monodromy matrices of $Y_{ \pm}^{\curvearrowleft}, Y_{ \pm}^{\bullet}$, around the points $x_{1, \pm}(\epsilon), x_{2, \pm}(\epsilon), \epsilon \epsilon$ $\mathrm{E}_{ \pm} \backslash\{0\}$, are given respectively by

$$
\begin{array}{ll}
M_{1, \pm}^{\bullet}=N_{x_{2, \pm}}^{(-1)} S_{1, \pm} N, & M_{2, \pm}^{\boldsymbol{\bullet}}=S_{2, \pm} N_{x_{2, \pm}} \\
M_{1, \pm}^{\bullet}=S_{1, \pm} N_{x_{1, \pm}}, & M_{2, \pm}^{\bullet}=N_{x_{2, \pm}} S_{2, \pm},
\end{array}
$$

where $S_{i, \pm}$ are of the form

$$
S_{1, \pm}=\left(\begin{array}{cc}
1 & 0 \\
s_{1, \pm} & 1
\end{array}\right), \quad S_{2, \pm}=\left(\begin{array}{cc}
1 & s_{2, \pm} \\
0 & 1
\end{array}\right)
$$

In particular $M_{1, \pm}^{\bullet}$ is lower-triangular and $M_{2, \pm}^{\bullet}$ is upper-triangular.

When $\epsilon \rightarrow 0$ along a sequence ${ }^{6} \frac{1}{\epsilon_{n}}=\frac{1}{\epsilon_{0}}+\frac{n}{\lambda^{(0)}}, n \in \pm \mathbb{N}, \epsilon_{0} \in \mathrm{E}_{ \pm} \backslash\{0\}$, these monodromy converge respectively to $\tilde{M}_{i, \pm}^{\bullet}(\kappa)=\lim _{n \rightarrow \pm \infty} M_{i, \pm}^{\bullet}\left(\epsilon_{n}\right)$ given by

$$
\begin{array}{llrl}
\tilde{M}_{1,+}^{\curvearrowleft}(\kappa)=\tilde{N}_{\epsilon}(\kappa)^{-1} S_{1}(0) N(0), & & \tilde{M}_{1,+}^{\bullet}(\kappa)=S_{1}(0) \tilde{N}_{0}(\kappa), \\
\tilde{M}_{2,+}^{\curvearrowleft}(\kappa)=S_{2}(0) \tilde{N}_{\epsilon}(\kappa), & & \tilde{M}_{2,+}^{\bullet}(\kappa)=\tilde{N}_{\epsilon}(\kappa) S_{2}(0), \\
\tilde{M}_{1,-}^{\curvearrowleft}(\kappa)=\tilde{N}_{0}(\kappa)^{-1} S_{1}(0) N(0), & & \tilde{M}_{1,-}^{\bullet}(\kappa)=S_{1}(0) \tilde{N}_{\epsilon}(\kappa), \\
\tilde{M}_{2,-}^{\curvearrowleft}(\kappa)=S_{2}(0) \tilde{N}_{0}(\kappa), & & \tilde{M}_{2,-}^{\bullet}(\kappa)=\tilde{N}_{0}(\kappa) S_{2}(0),
\end{array}
$$

with

$$
\begin{aligned}
\tilde{N}_{0}(\kappa) & =\left(\begin{array}{ll}
\kappa^{-1} & \\
& \kappa
\end{array}\right) T_{-2 \pi i \frac{d \lambda^{(0)}}{d \epsilon}(0)}, \quad \tilde{N}_{\epsilon}(\kappa)=\left(\begin{array}{ll}
\kappa & \\
& \kappa^{-1}
\end{array}\right) T_{2 \pi i\left[\frac{d \lambda^{(0)}}{d \epsilon}(0)+\lambda^{(1)}(0)\right]}, \\
N(0) & =T_{2 \pi i \lambda^{(1)}(0)} .
\end{aligned}
$$

We call them wild monodromy matrices. Their family

$$
\left\{\tilde{M}_{1, \pm}^{\bullet}(\kappa), \tilde{M}_{2, \pm}^{\bullet}(\kappa) \mid \kappa \in \mathbb{C}^{*}\right\}
$$

\footnotetext{
4 The name "wild monodromy" is borrowed from [19].

${ }^{5}$ For general linear systems one would need to add also the formal outer monodromy $N(0)=T_{2 \pi i \lambda(1)}(0)$, which in our case already belongs to the exponential torus.

6 The idea of taking limits of monodromy along such sequences can be found in the works of Ramis [23] or Duval [7].
} 
generates the same group, the representation of the wild monodromy group with respect to the formal solution $\hat{Y}(x, \epsilon)$, as does the collection of the Stokes matrices and the linear exponential torus

$$
\left\{S_{1}(0), S_{2}(0)\right\} \cup\left\{T_{a} \mid a \in \mathbb{C}\right\} .
$$

Hence we have the following theorem, whose general idea was suggested by Ramis [23]:

Theorem 42 When $\epsilon \rightarrow 0$ the elements of the monodromy group of the system (53) accumulate to generators of the wild monodromy group of the limit system.

\section{Confluent Degeneration of the Sixth Painlevé Equation to the Fifth}

The sixth Painlevé equation is

$$
\begin{aligned}
P_{V I}: q^{\prime \prime}= & \frac{1}{2}\left(\frac{1}{q}+\frac{1}{q-1}+\frac{1}{q-t}\right)\left(q^{\prime}\right)^{2}-\left(\frac{1}{t}+\frac{1}{t-1}+\frac{1}{q-t}\right) q^{\prime} \\
& +\frac{q(q-1)(q-t)}{2 t^{2}(t-1)^{2}}\left[\left(\vartheta_{\infty}-1\right)^{2}-\vartheta_{0}^{2} \frac{t}{q^{2}}+\vartheta_{1}^{2} \frac{(t-1)}{(q-1)^{2}}\right. \\
& \left.+\left(1-\vartheta_{t}^{2}\right) \frac{t(t-1)}{(q-t)^{2}}\right],
\end{aligned}
$$

where $\vartheta=\left(\vartheta_{0}, \vartheta_{t}, \vartheta_{1}, \vartheta_{\infty}\right) \in \mathbb{C}^{4}$ are complex constants. It is a reduction to the $q$-variable of a time dependent Hamiltonian system [21]

$$
\frac{d q}{d t}=\frac{\partial H_{V I}(q, p, t)}{\partial p}, \quad \frac{d p}{d t}=-\frac{\partial H_{V I}(q, p, t)}{\partial q},
$$

with a polynomial Hamiltonian function

$$
\begin{aligned}
H_{V I}= & \frac{1}{t(t-1)}\left[q(q-1)(q-t) p^{2}\right. \\
& -\left(\vartheta_{0}(q-1)(q-t)+\vartheta_{1} q(q-t)+\left(\vartheta_{t}-1\right) q(q-1)\right) p \\
& \left.+\frac{\left(\vartheta_{0}+\vartheta_{1}+\vartheta_{t}-1\right)^{2}-\vartheta_{\infty}^{2}}{4}(q-t)\right] .
\end{aligned}
$$

It has three simple (regular) singular points on the Riemann sphere $\mathbb{C P}^{1}$ at $t=0,1, \infty$.

The fifth Painlevé equation $P_{V}{ }^{7}$

$$
P_{V}: \quad q^{\prime \prime}=\left(\frac{1}{2 q}+\frac{1}{q-1}\right)\left(q^{\prime}\right)^{2}-\frac{1}{\tilde{t}} q^{\prime}+\frac{(q-1)^{2}}{2 \tilde{t}^{2}}\left(\left(\vartheta_{\infty}-1\right)^{2} q-\frac{\vartheta_{0}^{2}}{q}\right)
$$

\footnotetext{
7 The Eq. (6) is the fifth equation of Painlevé with a parameter $\eta_{1}=-1$. A general form of this equation would be obtained by a further change of variable $\tilde{t} \mapsto-\eta_{1} \tilde{t}$. The degenerate case $P_{V}^{\text {deg }}$ with $\eta_{1}=0$ which has only a regular singular point at $\infty$ is not considered here.
} 


$$
+\left(1+\tilde{\vartheta}_{1}\right) \frac{q}{\tilde{t}}-\frac{q(q+1)}{2(q-1)}
$$

is obtained from $P_{V I}$ as a limit $\epsilon \rightarrow 0$ after the change of the independent variable

$$
t=1+\epsilon \tilde{t}, \quad \vartheta_{t}=\frac{1}{\epsilon}, \quad \vartheta_{1}=-\frac{1}{\epsilon}+\tilde{\vartheta}_{1}+1,
$$

which sends the three singularities to $\tilde{t}=-\frac{1}{\epsilon}, 0, \infty$. At the limit, the two simple singular points $-\frac{1}{\epsilon}$ and $\infty$ merge into a double (irregular) singularity at the infinity.

The change of variables (58), changes the function $\epsilon \cdot H_{V I}$ to

$$
\begin{aligned}
H_{V I, \epsilon}= & \frac{1}{\tilde{t}(1+\epsilon \tilde{t})}\left[q(q-1)(q-1-\epsilon \tilde{t}) p^{2}\right. \\
& -\left(\vartheta_{0}(q-1)(q-1-\epsilon \tilde{t})+\tilde{\vartheta}_{1} q(q-1-\epsilon \tilde{t})+\tilde{t} q-\epsilon \tilde{t} q\right) p \\
& \left.+\frac{\left(\vartheta_{0}+\tilde{\vartheta}_{1}\right)^{2}-\vartheta_{\infty}^{2}}{4}(q-1-\epsilon \tilde{t})\right]
\end{aligned}
$$

and the Hamiltonian system to

$$
\frac{d q}{d \tilde{t}}=\frac{\partial H_{V I, \epsilon}(q, p, \tilde{t})}{\partial p}, \quad \frac{d p}{d \tilde{t}}=-\frac{\partial H_{V I, \epsilon}(q, p, \tilde{t})}{\partial q},
$$

whose limit $\epsilon \rightarrow 0$ is a Hamiltonian system of $P_{V}$. In the coordinate $x=\frac{1}{\tilde{t}}+\epsilon$, the above system is written as

$$
x(x-\epsilon) \frac{d q}{d x}=\frac{\partial H(q, p, x, \epsilon)}{\partial p}, \quad x(x-\epsilon) \frac{d p}{d x}=-\frac{\partial H(q, p, x, \epsilon)}{\partial q},
$$

with

$$
\begin{aligned}
H(q, p, x, \epsilon)= & -(1+\epsilon \tilde{t}) H_{V I, \epsilon}(q, p, \tilde{t}) \\
= & -\frac{\left(\vartheta_{0}+\tilde{\vartheta}_{1}\right)^{2}-\vartheta_{\infty}^{2}}{4}((x-\epsilon) q-x) \\
& +x \vartheta_{0} p+\left(1-\epsilon-(x-\epsilon) \vartheta_{0}-x\left(\vartheta_{0}+\tilde{\vartheta}_{1}\right)\right) q p \\
& +(2 x-\epsilon)(q p)^{2}+(x-\epsilon)\left(\vartheta_{0}+\tilde{\vartheta}_{1}\right) q^{2} p-x q p^{2}-(x-\epsilon) q^{3} p^{2}
\end{aligned}
$$

and Theorem 17 can be applied.

Theorem 43 The formal invariant $\chi$ of the system (59) is

$$
\chi(h, x, \epsilon)=1-\epsilon-(x-\epsilon) \vartheta_{0}-x\left(\vartheta_{0}+\tilde{\vartheta}_{1}\right)+2(2 x-\epsilon) h .
$$

Proof Let

$$
\tilde{q}=q-x A, \quad A=\frac{\vartheta_{0}}{1+\epsilon\left(1+\vartheta_{0}+\tilde{\vartheta}_{1}\right)},
$$




$$
\tilde{p}=p+(x-\epsilon) B, \quad B=\frac{\left(\vartheta_{0}+\tilde{\vartheta}_{1}\right)^{2}-\vartheta_{\infty}^{2}}{4\left(1+\epsilon\left(1-\vartheta_{0}\right)\right)}
$$

and let

$$
\tilde{H}(\tilde{q}, \tilde{p}, x, \epsilon)=H(q, p, x, \epsilon)-\frac{\left(\vartheta_{0}+\tilde{\vartheta}_{1}\right)^{2}+\vartheta_{\infty}^{2}}{4 t} x
$$

Then for $\epsilon \neq 0$,

$$
\tilde{H}(\tilde{q}, \tilde{p}, \epsilon, \epsilon)=\left(1-\epsilon\left(1+\vartheta_{0}+\tilde{\vartheta}_{1}\right)\right) \tilde{q} \tilde{p}+\epsilon(\tilde{q}+\epsilon A)^{2} \tilde{p}^{2}-\epsilon(\tilde{q}+\epsilon A) \tilde{p}^{2},
$$

hence by Proposition 9 the Birkhoff-Siegel invariant of $H(q, p, \epsilon, \epsilon)$ is

$$
G(h, \epsilon, \epsilon)=\left(1-\epsilon\left(1+\vartheta_{0}+\tilde{\vartheta}_{1}\right)\right) h+\epsilon h^{2},
$$

and

$$
\begin{aligned}
\tilde{H}(\tilde{q}, \tilde{p}, 0, \epsilon)= & \left(1-\epsilon\left(1-\vartheta_{0}\right)\right) \tilde{q} \tilde{p}-\epsilon \tilde{q}^{2}(\tilde{p}+\epsilon B)^{2}-\epsilon\left(\vartheta_{0}+\tilde{\vartheta}_{1}\right) \tilde{q}^{2}(\tilde{p}+\epsilon B) \\
& +\epsilon \tilde{q}^{3}(\tilde{p}+\epsilon B)^{2},
\end{aligned}
$$

hence by Proposition 9 the Birkhoff-Siegel invariant of $H(q, p, 0, \epsilon)$ is

$$
G(h, 0, \epsilon)=\left(1-\epsilon\left(1-\vartheta_{0}\right)\right) h-\epsilon h^{2},
$$

i.e.

$$
G(h, x, \epsilon)=\left(1-\epsilon-(x-\epsilon) \vartheta_{0}-x\left(\vartheta_{0}+\tilde{\vartheta}_{1}\right)\right) h+(2 x-\epsilon) h^{2}
$$

The Theorem 16 for the limit system $\epsilon=0$ is in this case due to Takano [30], see also [27,35]. A separate paper [14] will be devoted to a more detailed study of the confluence $P_{V I} \rightarrow P_{V}$ and of the non-linear Stokes phenomenon in $P_{V}$ through the Riemann-Hilbert correspondence.

\section{Proof of Theorem 17 and of Proposition 9}

The proof of Theorem 17 is loosely based on the ideas of Siegel's proof of Theorem 4 [28, chaps. 16 and 17], using similar tools as [4]. We construct the normalizing transformation $y=\Phi_{ \pm}(u, x, \epsilon)$ in a couple of steps as a formal power series in the $u$-variable with coefficients depending analytically on $(x, \epsilon) \in \mathrm{XE}_{ \pm}$, and then show that the series is convergent. The main tool to prove the convergence is the Lemma 44 below for series with coefficients in a Banach algebra.

Let $(\mathfrak{B},\|\cdot\|)$ be a Banach algebra, and let

$$
\phi(u)=\sum_{|\mathbf{m}| \geq 2} \phi_{\mathbf{m}} u^{\mathbf{m}}, \quad \mathbf{m}=\left(m_{1}, m_{2}\right), u^{\mathbf{m}}=u_{1}^{m_{1}} u_{2}^{m_{2}},|\mathbf{m}|=m_{1}+m_{2},
$$


be a formal power series in the $u$-variable with coefficients $\phi_{\mathbf{m}} \in \mathfrak{B}$, and denote

$$
\bar{\phi}(u)=\sum_{|\mathbf{m}| \geq 2}\left\|\phi_{\mathbf{m}}\right\| u^{\mathbf{m}}
$$

its majorant power series. We will use the notation

$$
\{\phi\}_{\mathbf{m}}:=\phi_{\mathbf{m}},
$$

and

$$
\bar{\phi}(u) \prec \bar{\psi}(u) \text { if }\left\|\{\phi\}_{\mathbf{m}}\right\| \leq\left\|\{\psi\}_{\mathbf{m}}\right\| \text { for all } \mathbf{m} \text {. }
$$

Lemma 44 Let $(\mathfrak{B},\|\cdot\|)$ be a Banach algebra, let $\phi={ }^{t}\left(\phi_{1}, \phi_{2}\right)=O\left(u^{2}\right)$ be a formal power series in $u$ with coefficients in $\mathfrak{B}^{2}$, and let $r={ }^{\mathrm{t}}\left(r_{1}, r_{2}\right)=O\left(u^{2}\right)$ be a power series in $u$ with coefficients in $\mathfrak{B}^{2}$ convergent w.r.t. the norm $\|\cdot\|$. If

$$
\bar{\phi}_{j}(u) \prec \bar{r}_{j}(u+\bar{\phi}(u)), \quad j=1,2,
$$

where $\bar{\phi}={ }^{\mathrm{t}}\left(\bar{\phi}_{1}, \bar{\phi}_{2}\right)$, then $\phi$ is convergent w.r.t. the norm $\|\cdot\|$.

Proof See [24, Theorem 2.2] - the generalization to Banach algebra setting comes for free with no change in the proof. This lemma can also be implicitly found in [20, p.520] and in [28, chap. 17] as the technique used in Siegel's proof of Theorem 4.

We will introduce two Banach algebras: (i) an algebra $\mathfrak{B}\left(X E_{ \pm}\right)$of bounded analytic functions on the domain $X E_{ \pm}$, and (ii) an algebra $\hat{\mathfrak{B}}\left(\Omega_{ \pm}^{0}\right)$ of certain Borel summable formal power series in $(x, \epsilon)$, with the goal to construct the sectoral normalizing transformation $\Psi_{ \pm}(u, x, \epsilon)$, resp. the formal normalizing transformation $\hat{\Psi}(u, x, \epsilon)$, of Theorem 17 as a convergent power series of $u$ in the algebra $\mathfrak{B}\left(X E_{ \pm}\right)$, resp. $\hat{\mathfrak{B}}\left(\Omega_{ \pm}^{0}\right)$.

Banach algebra of bounded analytic functions $\mathfrak{B}\left(\mathrm{XE}_{ \pm}\right)$. We denote $\mathfrak{B}\left(\mathrm{XE}_{ \pm}\right)$the space of all such functions $f: \mathbf{X E}_{ \pm} \rightarrow \mathbb{C}$ that are bounded and uniformly continuous on the ramified domain $X E_{ \pm}(25)$ and analytic on its interior. The space $\mathfrak{B}\left(\mathrm{XE}_{ \pm}\right)$ endowed with the supremum norm

$$
\|f\|:=\sup _{(x, \epsilon) \in \mathrm{XE}_{ \pm}}|f(x, \epsilon)|
$$

clearly forms a Banach algebra.

Banach algebra of Borel $(1,1)$-summable formal power series $\hat{\mathfrak{B}}\left(\Omega_{ \pm}^{0}\right)$. Next we introduce a type of Borel summation procedure for certain formal series of $(x, \epsilon)$ following [2], and provide a suitable norm which makes the space of them into a Banach algebra. While the construction is a bit technical, the details of it won't be needed beyond this section.

For $\theta \in]-\frac{\pi}{2}, \frac{\pi}{2}[$ let us denote

$$
\mathbb{B}_{\theta}:=\left\{\sigma \in \mathbb{C}: \Re\left(\frac{e^{i \theta}}{\sigma}\right) \geq \cos \theta\right\} \cup\{0\}
$$


the closed disc with center on $e^{i \theta} \mathbb{R}^{+}$and the points 0 and 1 on its circumference.

Let $r>0$ be a small constant which will appear in Theorem 47 below. We will consider formal power series

$$
\hat{f}(x, \epsilon)=\sum_{k, l \geq 0} f^{(k, l)} x^{k} \epsilon^{l}, \quad \text { such that } \sup _{k, l \geq 0}\left|f^{(k, l)}\right| \frac{r^{k+l}}{(k+l) !}<+\infty,
$$

i.e. such that the transformed series

$$
\widetilde{f}(x, \epsilon):=\sum_{k, l} \frac{f^{(k, l)}}{(k+l) !} x^{k} \epsilon^{l},
$$

converges on the polydisc $D_{r}=\{|x|,|\epsilon|<r\}$. Supposing that $\tilde{f}$ extends analytically on a ray $\left(x_{0}, \epsilon_{0}\right) \cdot \mathbb{R}^{+}$for some $\left(x_{0}, \epsilon_{0}\right)$ and has at most exponential growth there: $\int_{0}^{+\infty}\left|\tilde{f}\left(s x_{0}, s \epsilon_{0}\right)\right| e^{-\Lambda\left(x_{0}, \epsilon_{0}\right) s} d s<+\infty$ for some $\Lambda\left(x_{0}, \epsilon_{0}\right)>0$, then the following Laplace transform of $\tilde{f}$ :

$$
\mathcal{L}_{0}[\tilde{f}](x, \epsilon):=\int_{0}^{+\infty} \widetilde{f}(s x, s \epsilon) e^{-s} d s,
$$

is convergent on the segment $(x, \epsilon) \in\left(x_{0}, \epsilon_{0}\right) \cdot\left[0, \Lambda\left(x_{0}, \epsilon_{0}\right)\right]$. The function (62) extends analytically as a bounded function to the "Borel disc" $\left(x_{0}, \epsilon_{0}\right) \cdot \Lambda\left(x_{0}, \epsilon_{0}\right) \mathbb{B}_{0}$, by

$$
\mathcal{L}_{\theta}[\tilde{f}](x, \epsilon):=\int_{0}^{+\infty e^{i \theta}} \tilde{f}(s x, s \epsilon) e^{-s} d s,
$$

where $\theta \in]-\frac{\pi}{2}, \frac{\pi}{2}\left[\right.$ depending on $(x, \epsilon)$ is such that $(x, \epsilon) \cdot e^{i \theta} \in\left(x_{0}, \epsilon_{0}\right) \mathbb{R}^{+}$. The function (62) is called the Borel $(1,1)$-sum of $\hat{f}(x, \epsilon)$ in the direction $\left(x_{0}, \epsilon_{0}\right)$ [2], and satisfies the same $\left(\partial_{x}, \partial_{\epsilon}\right)$-differential relations over the ring of convergent power series as $\hat{f}(x, \epsilon)$.

We shall now define a space $\hat{\mathfrak{B}}\left(\Omega_{ \pm}^{0}\right)$ of thus summable power series, and a suitable norm that would make it into a Banach algebra.

\section{Definition 45 Let}

$$
\Omega_{ \pm}^{0}:=\left\{(x, \epsilon) \in \mathrm{XE}_{ \pm}:(x, \epsilon) \cdot \mathbb{B}_{0} \subseteq \mathrm{XE}_{ \pm}\right\} \subseteq \mathrm{XE}_{ \pm}
$$

where $\mathbb{B}_{0}$ is the disc (61), and let $r>0$ as before. Let

$$
\Lambda(x, \epsilon):=\max \left\{\frac{|x|}{\delta_{x}}, \frac{|\epsilon|}{\delta_{\epsilon}}\right\}
$$

where $\delta_{x}, \delta_{\epsilon}>0$ are such as in Definition 12. Let us consider formal power series $\hat{f}(x, \epsilon)=\sum_{k, l \geq 0} f^{(k, l)} x^{k} \epsilon^{l}$ such that the transformed series $\widetilde{f}(x, \epsilon)=$ $\sum_{k, l} \frac{f^{(k, l)}}{(k+l) !} x^{k} \epsilon^{l}$, is convergent on the polydisc $D_{r}=\{|x|,|\epsilon|<r\}$ and extends analytically on the cone $\Omega_{ \pm}^{0} \cdot \mathbb{R}^{+}$. We introduce a norm of the formal series $\hat{f}(x, \epsilon)$, based on 
similar norms in $[5,6]$, as an exponentially weighted supremum norm of the function $\left(x \partial_{x}+\epsilon \partial_{\epsilon}\right) \tilde{f}:$

$$
\|\hat{f}\|_{\Lambda}:=\sup _{\substack{(x, \epsilon) \in \in \\ D_{r} \cup \Omega_{ \pm}^{0} \cdot \mathbb{R}^{+}}} \frac{4 \pi}{\Lambda(x, \epsilon)}\left|\left(x \partial_{x}+\epsilon \partial_{\epsilon}\right) \tilde{f}(x, \epsilon)\right| e^{-\Lambda(x, \epsilon)}\left(1+\Lambda(x, \epsilon)^{2}\right),
$$

and define $\hat{\mathfrak{B}}\left(\Omega_{ \pm}^{0}\right)$ as the space of such $\hat{f}$ for which $\|\hat{f}\|_{\Lambda}<+\infty$.

If $\hat{f} \in \hat{\mathfrak{B}}\left(\Omega_{ \pm}^{0}\right)$, then its $\operatorname{Borel}(1,1)$-sum $f_{ \pm}(x, \epsilon)$ defined through the Laplace integral (62) converges for every $(x, \epsilon) \in \Omega_{ \pm}^{0}$ with $\Lambda(x, \epsilon)<1$ (which is automatically satisfied), and it extends analytically as a bounded function on

$$
\Omega_{ \pm}=\bigcup_{(x, \epsilon) \in \Omega_{ \pm}^{0}}(x, \epsilon) \cdot \mathbb{B}_{0}=\left\{(x, \epsilon) \in \mathrm{XE}_{ \pm}, \exists \theta \in\right]-\frac{\pi}{2}, \frac{\pi}{2}\left[:(x, \epsilon) \cdot \mathbb{B}_{\theta} \subset \mathrm{XE}_{ \pm}\right\}
$$

Note that if a sequence of formal series in $\hat{\mathfrak{B}}\left(\Omega_{ \pm}^{0}\right)$ converges with respect to the norm $\|\cdot\|_{\Lambda}$ then they also converge in the product topology on $\mathbb{C}[[x, \epsilon]]$, i.e. the coefficients of the power series converge term by term, and moreover the sequence of the corresponding Borel (1,1)-sums converges uniformly on compacts of $\Omega_{ \pm}$. In particular, if $\sum_{\mathbf{m}} \hat{\phi}_{\mathbf{m}}(x, \epsilon) u^{\mathbf{m}}, \hat{\phi}_{\mathbf{m}}(x, \epsilon)=\sum_{k, l \geq 0} \phi_{\mathbf{m}}^{(k, l)} x^{k} \epsilon^{l} \in \hat{\mathfrak{B}}\left(\Omega_{ \pm}^{0}\right)$, is a convergent power series in the Banach space $\left(\hat{\mathfrak{B}}\left(\Omega_{ \pm}^{0}\right),\|\cdot\|_{\Lambda}\right)$ for $u \in \mathrm{U}$, then its sum is a map $U \rightarrow \hat{\mathfrak{B}}\left(\Omega_{ \pm}^{0}\right)$ given by a formal series $\hat{\phi}(u, x, \epsilon)=\sum_{k, l \geq 0} \phi^{(k, l)}(u) x^{k} \epsilon^{l}$, with each $\phi^{(k, l)}(u)=\sum_{\mathbf{m}} \phi_{\mathbf{m}}^{(k, l)} u^{\mathbf{m}}$ convergent on $\mathrm{U}$. Moreover, it follows that the Borel $(1,1)$-sum of $\hat{\phi}(u, x, \epsilon)$, given by the Laplace integral $\mathcal{L}_{0}[\widetilde{\phi}(u, \cdot, \cdot)](x, \epsilon)(62)$, with $\widetilde{\phi}(u, x, \epsilon)=\sum_{k, l \geq 0} \frac{\phi^{(k, l)}(u)}{(k+l) !} x^{k} \epsilon^{l}$, agrees with the convergent series of the Borel $(1,1)$-sums $\sum_{\mathbf{m}} \mathcal{L}_{0}\left[\widetilde{\phi}_{\mathbf{m}}\right](x, \epsilon) u^{\mathbf{m}}$, which can be showed by a dominated convergence argument.

Proposition 46 The space $\left(\hat{\mathfrak{B}}\left(\Omega_{ \pm}^{0}\right),\|\cdot\|_{\Lambda}\right)$ is a Banach algebra.

Proof It is quite clear that $\left(\hat{\mathfrak{B}}\left(\Omega_{ \pm}^{0}\right),\|\cdot\|_{\Lambda}\right)$ is a Banach space. What rests is to show the sub-multiplicativity property of the norm:

$$
\|\hat{f} \hat{g}\|_{\Lambda} \leq\|\hat{f}\|_{\Lambda}\|\hat{g}\|_{\Lambda} \quad \text { for all } \hat{f}, \hat{g} \in \hat{\mathfrak{B}}\left(\Omega_{ \pm}^{0}\right)
$$

Given $\hat{f}(x, \epsilon) \in \hat{\mathfrak{B}}\left(\Omega_{ \pm}^{0}\right)$, consider the formal series

$$
\hat{F}(t, x, \epsilon)=\hat{f}(t x, t \epsilon)=\sum_{k, l \geq 0} f^{(k, l)} x^{k} \epsilon^{l} t^{k+l}=\sum_{n \geq 0}\left(\sum_{k+l=n} f^{(k, l)} x^{k} \epsilon^{l}\right) t^{n}
$$


of a new variable $t$ with parameters $(x, \epsilon)$. The usual Borel transform of $\hat{F}$ with respect to $t$ is given by

$$
\mathcal{B}[\hat{F}](s, x, \epsilon):=\sum_{k, l \geq 0} \frac{f^{(k, l)}}{(k+l-1) !} x^{k} \epsilon^{l} s^{k+l-1}=\partial_{s} \tilde{f}(s x, s \epsilon) .
$$

Hence

$$
\|\hat{f}\|_{\Lambda}=\sup _{(s x, s \epsilon) \in D_{r} \cup \Omega_{ \pm}^{0} \cdot \mathbb{R}^{+}} \frac{4 \pi}{\Lambda(x, \epsilon)}|\mathcal{B}[\hat{F}](s, x, \epsilon)| e^{-\Lambda(x, \epsilon)|s|}\left(1+\Lambda(x, \epsilon)^{2}|s|^{2}\right),
$$

which is a rescaling of the weighted supremum norm introduced in [5, p.312]. The usual Borel transform $\mathcal{B}$ changes products into convolutions,

$$
\mathcal{B}[\hat{F} \hat{G}](t, x, \epsilon)=(\mathcal{B}[\hat{F}] * \mathcal{B}[\hat{G}])(t, x, \epsilon):=\int_{0}^{t} \mathcal{B}[\hat{F}](t-s, x, \epsilon) \mathcal{B}[\hat{G}](s, x, \epsilon) d s
$$

and we can conclude by the estimate of a norm of convolution in [5, Proposition 4] which gives us the result.

The reason for introducing the two Banach algebras the above way lies in the following theorem adapted from [13] which is the other essential tool in our proof of Theorem 17.

Theorem 47 Consider a system of the form

$$
x(x-\epsilon) \frac{d \phi}{d x}=M \phi+F(\phi, x, \epsilon), \quad(\phi, x, \epsilon) \in \mathbb{C}^{m} \times \mathbb{C} \times \mathbb{C} .
$$

with $M$ an invertible $m \times m$-matrix whose eigenvalues all lie on the line $\lambda^{(0)} \mathbb{R}$, and $F(\phi, x, \epsilon)$ analytic germ such that $D_{\phi} F(0,0,0)=0$, and $F(0, x, \epsilon)=O(x(x-\epsilon))$. Then there exist some constants $r, \eta, \delta_{x}, \delta_{\epsilon}>0$ such that the system (65) possesses:

(1) A unique formal power series solution $\hat{\phi}(x, \epsilon)=\sum_{j, k \geq 0} \phi_{k j} x^{k} \epsilon^{j}$. It is formally divisible by $x(x-\epsilon)$, and it belongs to $\left(\hat{\mathfrak{B}}\left(\Omega_{ \pm}^{0}\right)\right)^{m}$.

(2) A unique bounded analytic solution $\phi_{ \pm}(x, \epsilon) \in\left(\mathfrak{B}\left(\mathrm{XE}_{ \pm}\right)\right)^{m}$ on the domain $\mathrm{XE}_{ \pm}$ of Definition 12. It vanishes at the singular points (it is uniformly $O(x(x-\epsilon))$ ).

Moreover, the restriction of $\phi_{ \pm}(x, \epsilon)$ to the domain $\Omega_{ \pm}$agrees with the Borel $(1,1)$ sum of the formal solution $\hat{\phi}(x, \epsilon)$ :

$$
\phi_{ \pm}(x, \epsilon)=\int_{0}^{+\infty e^{i \theta}} \widetilde{\phi}(s x, s \epsilon) e^{-s} d s, \quad \text { for } \quad(x, \epsilon) \in \Omega_{ \pm}^{0},
$$

where $\theta \in]-\frac{\pi}{2}, \frac{\pi}{2}\left[\right.$ depending on $(x, \epsilon)$ is such that $(x, \epsilon) \cdot \mathbb{B}_{\theta} \subset \mathrm{XE}_{ \pm}$, and $\widetilde{\phi}(x, \epsilon)=$ $\sum_{j, k} \frac{\phi_{k j}}{(k+j) !} x^{k} \epsilon^{j}$. 
Proof The existence and uniqueness of the bounded solution $\phi_{ \pm}(x, \epsilon)$ is [13, Theorem 2]. The existence and uniqueness of the formal solution $\hat{\phi}(x, \epsilon)$ is [13, Proposition 1], while the Borel (1,1)-summability is [13, Theorem 4]. The finiteness of the norm $\|\hat{\phi}\|_{\Lambda}$ is just a consequence of its Borel $(1,1)$-summability, up to perhaps restricting of $\delta_{x}, \delta_{\epsilon}>0$.

\subsection{Step 1: Ramified Straightening of Center Manifold and Diagonalization of the Linear Part}

Suppose that the system is in a pre-normal form,

$$
\begin{aligned}
& x(x-\epsilon) \frac{d y}{d x}=J{ }^{\mathrm{t}} D_{y} F(y, x, \epsilon), \\
& J^{\mathrm{t}} D_{y} F(y, x, \epsilon)=\chi\left(y_{1} y_{2}, x, \epsilon\right)\left(\begin{array}{cc}
1 & 0 \\
0 & -1
\end{array}\right) y+O(x(x-\epsilon)) .
\end{aligned}
$$

We will show that there exists a ramified transversely symplectic change of variable

$$
y=T_{ \pm}(x, \epsilon) w+\phi_{ \pm}(x, \epsilon), \quad \operatorname{det} T_{ \pm}(0, \epsilon)=I, \phi_{ \pm}(x, \epsilon)=O(x(x-\epsilon))
$$

bounded and analytic on the domain $\mathrm{XE}_{ \pm}(25)$, i.e. defined over the Banach space $\mathfrak{B}\left(\mathrm{XE}_{ \pm}\right)$, that brings the system to a form

$$
x(x-\epsilon) \frac{d w}{d x}=\chi\left(w_{1} w_{2}, x, \epsilon\right)\left(\begin{array}{cc}
1 & 0 \\
0 & -1
\end{array}\right) w+x(x-\epsilon) f_{ \pm}(w, x, \epsilon),
$$

with $f_{ \pm}(w, x, \epsilon)=O\left(|w|^{2}\right), \frac{\partial f_{1, \pm}}{\partial w_{1}}+\frac{\partial f_{2, \pm}}{\partial w_{2}}=0$.

All this has a formal counterpart over the Banach space $\hat{\mathfrak{B}}\left(\Omega_{ \pm}^{0}\right)$.

The solution $w=0$ of the transformed system (69), corresponds to a bounded ramified solution $y=\phi_{ \pm}(x, \epsilon)$ of the system (67). The paper [13], see Theorem 47 below, shows that there is a unique such solution on the domain $\mathrm{XE}_{ \pm}$; this it is the "ramified center manifold" of the corresponding foliation.

The variable $\tilde{y}=y-\phi_{ \pm}(x, \epsilon)$ then satisfies

$$
x(x-\epsilon) \frac{d \tilde{y}}{d x}=J{ }^{\mathrm{t}} D_{y} F\left(\tilde{y}+\phi_{ \pm}, x, \epsilon\right)-J{ }^{\mathrm{t}} D_{y} F\left(\phi_{ \pm}, x, \epsilon\right),
$$

whose linear part is $A_{ \pm}(x, \epsilon):=J\left(D_{y}^{2} F\right)\left(\phi_{ \pm}, x, \epsilon\right)=\lambda(x, \epsilon)\left(\begin{array}{cc}1 & 0 \\ 0 & -1\end{array}\right)+x(x-$ $\epsilon) R\left(\phi_{ \pm}, x, \epsilon\right)$. The transformation matrix $T_{ \pm}$(68) must then satisfy

$$
x(x-\epsilon) \frac{d T_{ \pm}}{d x}=A_{ \pm} T_{ \pm}-\lambda T_{ \pm}\left(\begin{array}{cc}
1 & 0 \\
0 & -1
\end{array}\right) .
$$


The existence of such a transformation $T_{ \pm}$bounded on $\mathrm{XE}_{ \pm}$is known $[10,15]$ when $A_{ \pm}$is analytic. In our case the matrix $A_{ \pm}$is ramified, but their proof would work anyway. We will obtain $T_{ \pm}$directly using Theorem 47 .

Writing $R=\left(r_{i j}\right)_{i, j}$ and

$$
T_{ \pm}=\left(\begin{array}{cc}
1 & t_{1, \pm} \\
t_{2, \pm} & 1
\end{array}\right)\left(\begin{array}{cc}
e^{b_{1, \pm}} & 0 \\
0 & e^{b_{2, \pm}}
\end{array}\right)
$$

then the terms $t_{i, \pm}, i=1,2$, are solutions to Riccati equations

$$
x(x-\epsilon) \frac{d t_{i, \pm}}{d x}=(-1)^{i-1} 2 \lambda t_{i, \pm}+x(x-\epsilon)\left[r_{i j}+\left(r_{i i}-r_{j j}\right) \cdot t_{i, \pm}-r_{j i} \cdot\left(t_{i, \pm}\right)^{2}\right],
$$

and the terms $b_{i, \pm}$ are solution to $\frac{d b_{i, \pm}}{d x}=\left(r_{i i}+r_{i j} t_{j, \pm}\right)$, i.e.

$$
b_{i, \pm}=\int_{0}^{x} r_{i i}+r_{i j} t_{j, \pm} d x
$$

Combining together the equations (67) for $\phi_{ \pm}$and (70) for $t_{ \pm}$, in which $r_{i j}=$ $r_{i, j}\left(\phi_{ \pm}, x, \epsilon\right)$, we get an analytic system for which the existence of a unique bounded solution on $\mathrm{XE}_{ \pm}$is assured by Theorem 47 .

Since the trace of the linear part of both systems (67) and (69) is null, then by the Liouville-Ostrogradskii formula det $T(x, \epsilon)$ is constant in $x$ and equal to det $T(0, \epsilon)=$ 1. Therefore the transformation (68) is transversely symplectic, and by Lemma 3 the transformed system (69) is transversely Hamiltonian.

\subsection{Step 2: Normalization}

Suppose that the system is in the form (69). We will show that there exists a ramified change of variable $w=\Phi_{ \pm}(v, x, \epsilon), \Phi_{ \pm}(\cdot, 0, \epsilon)=\mathrm{id}$, given as a convergent series of $v$ in the Banach space $\mathfrak{B}\left(\mathrm{XE}_{ \pm}\right)$, that will bring it to an integrable form

$$
x(x-\epsilon) \frac{d v}{d x}=\alpha_{ \pm}(h, x, \epsilon)\left(\begin{array}{cc}
1 & 0 \\
0 & -1
\end{array}\right) v, \quad h=v_{1} v_{2}
$$

for some germ $\alpha_{ \pm}(h, x, \epsilon)$, with $\alpha_{ \pm}(0, x, \epsilon)=\lambda(x, \epsilon)$.

As before, this has a formal counterpart over the Banach space $\hat{\mathfrak{B}}\left(\Omega_{ \pm}^{0}\right)$.

The transformation $\Phi_{ \pm}$must satisfy

$$
x(x-\epsilon) \partial_{x} \Phi_{ \pm}+\alpha_{ \pm} \cdot\left(v_{1} \partial_{v_{1}}-v_{2} \partial_{v_{2}}\right) \Phi_{ \pm}=\chi \circ \Phi_{ \pm} \cdot\left(\begin{array}{cc}
1 & 0 \\
0 & -1
\end{array}\right) \Phi_{ \pm}+x(x-\epsilon) f_{ \pm} \circ \Phi_{ \pm} .
$$

We are looking for $\Phi_{ \pm}$written as

$$
\Phi_{ \pm}(v, x, \epsilon)=v+\Psi_{ \pm, \Delta}+x(x-\epsilon) \Psi_{ \pm, \star}(v, x, \epsilon), \quad \Psi_{ \pm, \Delta}+\Psi_{ \pm, \star}=: \Psi_{ \pm}
$$


where the power expansion of the $j$-th coordinate of $\Psi_{ \pm, \Delta}$ is equal to

$$
\Psi_{j, \pm, \Delta}=\sum_{n \geq 1}\left\{\Psi_{j, \pm}\right\}_{(n, n)+\mathbf{e}_{j}} h^{n} v_{j}, \quad \mathbf{e}_{j} \text { being the } j \text {-the elementary vector, }
$$

while the power expansion of the $j$-th coordinate of $\left\{\Psi_{ \pm, \star}\right\}$ does not contains any power $h^{n} v_{j}, n \geq 0$. In particular, $\left(v_{1} \partial_{v_{1}}-v_{2} \partial_{v_{2}}\right) \Psi_{ \pm, \Delta}=\left(\begin{array}{cc}1 & 0 \\ 0 & -1\end{array}\right) \Psi_{ \pm, \Delta}$. Therefore (72) becomes

$$
\begin{aligned}
\partial_{x} \Psi_{ \pm, \Delta}+\partial_{x}\left(x(x-\epsilon) \Psi_{ \pm, \star}\right)+\lambda\left(v_{1} \partial_{v_{1}}-v_{2} \partial_{v_{2}}-\left(\begin{array}{cc}
1 & 0 \\
0 & -1
\end{array}\right)\right) \Psi_{ \pm, \star} \\
=-\alpha_{ \pm}^{*}\left(v_{1} \partial_{v_{1}}-v_{2} \partial_{v_{2}}\right) \Psi_{ \pm, \star}+\frac{\chi^{*} \circ \Phi_{ \pm}-\alpha_{ \pm}^{*}}{x(x-\epsilon)}\left(\begin{array}{cc}
1 & 0 \\
0 & -1
\end{array}\right)\left(v+\Psi_{ \pm, \Delta}\right)+G_{ \pm}
\end{aligned}
$$

where $G_{ \pm}=\left(\chi^{*} \circ \Phi_{ \pm}\right)\left(\begin{array}{cc}1 & 0 \\ 0 & -1\end{array}\right) \Psi_{ \pm, \star}+f \circ \Phi_{ \pm}$, and $\chi^{*}=\chi-\lambda, \alpha_{ \pm}^{*}=\alpha_{ \pm}-\lambda$.

Set

$$
\alpha_{ \pm}(h, x, \epsilon)=\sum_{n \geq 0}\left\{\chi \circ \Phi_{ \pm}\right\}_{(n, n)} h^{n}
$$

and denote

$$
K_{ \pm}:=\frac{\chi^{*} \circ \Phi_{ \pm}-\chi^{*} \circ\left(v+\Psi_{ \pm, \Delta}\right)}{x(x-\epsilon)}
$$

which is an analytic function of $v+\Psi_{ \pm, \Delta}$ and $\Psi_{ \pm, \star}$ with coefficients depending on $x, \epsilon$. Then $\left\{\chi^{*} \circ \Phi_{ \pm}-\alpha_{ \pm}^{*}\right\}_{(n, n)}=0$ for all $n \geq 0$, and $\left\{\chi^{*} \circ \Phi_{ \pm}-\alpha_{ \pm}^{*}\right\}_{\mathbf{n}}=x(x-\epsilon)\left\{K_{ \pm}\right\}_{\mathbf{n}}$ for all multi-indices $\mathbf{n}$ with $n_{1} \neq n_{2}$, since $\left\{\alpha_{ \pm}^{*}\right\}_{\mathbf{n}}=0=\left\{\chi^{*} \circ\left(v+\Psi_{ \pm, \Delta}\right)\right\}_{\mathbf{n}}$.

Expanding the $j$-th coordinate, $j=1,2$, of the Eq. (72) in powers of $v$ we get:

- for $\mathbf{m}=(n, n)+\mathbf{e}_{j}$ :

$$
\partial_{x}\left\{\Psi_{j, \pm}\right\}_{(n, n)+\mathbf{e}_{j}}=\left\{G_{j, \pm}\right\}_{(n, n)+\mathbf{e}_{j}},
$$

- for a multi-index m with $m_{1}-m_{2}+(-1)^{j} \neq 0$ :

$$
\begin{aligned}
& \partial_{x}\left\{x(x-\epsilon) \Psi_{j, \pm}\right\}_{\mathbf{m}}+\left(m_{1}-m_{2}+(-1)^{j}\right) \lambda\left\{\Psi_{j, \pm}\right\}_{\mathbf{m}} \\
& =-\left(m_{1}-m_{2}\right)\left\{\alpha_{ \pm}^{*} \cdot \Psi_{j, \pm}\right\}_{\mathbf{m}}-(-1)^{j}\left\{K_{ \pm} \cdot\left(v_{j}+\Psi_{j, \pm, \Delta}\right)\right\}_{\mathbf{m}}+\left\{G_{j, \pm}\right\}_{\mathbf{m}} .
\end{aligned}
$$

The right-hand sides of (76) and (75) are functions of $\left\{\Psi_{ \pm}\right\}_{\mathbf{k}}={ }^{\mathrm{t}}\left(\left\{\Psi_{1, \pm}\right\}_{\mathbf{k}},\left\{\Psi_{2, \pm}\right\}_{\mathbf{k}}\right)$, with $k_{1} \leq m_{1}, k_{2} \leq m_{2},|\mathbf{k}|<|\mathbf{m}|$ only, which means that the equations for $\left\{\Psi_{j, \pm}\right\}_{\mathbf{m}}$ can be solved recursively.

The Eq. (75) is solved by

$$
\left\{\Psi_{j, \pm}\right\}_{(n, n)+\mathbf{e}_{j}}(x, \epsilon)=\int_{0}^{x}\left\{G_{j, \pm}\right\}_{(n, n)+\mathbf{e}_{j}} d x
$$


The Eq. (76) has a unique bounded solution $\left\{\Psi_{j, \pm}\right\}_{\mathbf{m}}$ given by the integral

$$
\left\{\Psi_{j, \pm}\right\}_{\mathbf{m}}(x, \epsilon)=\frac{e^{-\left(m_{1}-m_{2}+(-1)^{j}\right) t_{\lambda}}}{x(x-\epsilon)} \int_{x_{i, \pm}}^{x} e^{\left(m_{1}-m_{2}+(-1)^{j}\right) t_{\lambda}}\left\{F_{j, \pm}\right\}_{\mathbf{m}} d x
$$

where $\left\{F_{j, \pm}\right\}_{\mathbf{m}}$ is the right side of (76),

$$
t_{\lambda}(x, \epsilon)=\int_{\infty}^{x} \frac{\lambda(x, \epsilon)}{x(x-\epsilon)} d x= \begin{cases}-\frac{\lambda^{(0)}}{\epsilon} \log x+\left(\frac{\lambda^{(0)}}{\epsilon}+\lambda^{(1)}\right) \log (x-\epsilon), & \text { for } \epsilon \neq 0 \\ -\frac{\lambda^{(0)}}{x}+\lambda^{(1)} \log x, & \text { for } \epsilon=0\end{cases}
$$

is a branch of the rectifying coordinate for the vector field

$$
\frac{x(x-\epsilon)}{\lambda(x, \epsilon)} \partial_{x}=\partial_{t_{\lambda}}
$$

on $\mathrm{X}_{ \pm}(\epsilon)$, and the integration follows a real trajectory of the vector field (23) in $\mathrm{X}_{ \pm}(\epsilon)$ from a point

$$
x_{*}=\left\{\begin{array}{l}
x_{1, \pm}, \text { if } m_{1}-m_{2}+(-1)^{j}>0, \\
x_{2, \pm}, \text { if } m_{1}-m_{2}+(-1)^{j}<0,
\end{array} \quad x_{i, \pm}\right. \text { is as in (26), }
$$

to $x$, along which the integral is well defined.

Note that the convergence of the constructed formal transformation $\Phi_{ \pm}$is equivalent to the convergence of $\Psi_{ \pm}$(73). We prove the convergence of the latter series using Lemma 44. For this we need to estimate the norms of (77) and (78).

Lemma 48 Let $\left\{\Psi_{j, \pm}\right\}_{\mathbf{m}}$ be given by (78). Then

$$
\left\|\left\{\Psi_{j, \pm}\right\}_{\mathbf{m}}\right\| \leq \frac{c_{\star}}{m_{1}-m_{2}+(-1)^{j}}\left\|\left\{F_{j, \pm}\right\}_{\mathbf{m}}\right\|,
$$

for some $c_{\star}>0$ independent of $\mathbf{m}$.

Proof Let $\tau_{k}(x, \epsilon)=k t_{\lambda}(x, \epsilon)+\log (x(x-\epsilon))$. If $k=m_{1}-m_{2}+(-1)^{j} \neq 0$, then for $\epsilon$ small enough the integrating path can be deformed so that it corresponds to a ray $\tau_{k}(\xi, \epsilon) \in \tau_{k}(x, \epsilon)-e^{i \theta_{ \pm}}\left[0,+\infty\left[\right.\right.$, with $\theta_{ \pm}$as in (24). We have $\frac{d \tau_{k}}{d x}(x, \epsilon)=$ $\frac{k \lambda(x, \epsilon)+2 x-\epsilon}{x(x-\epsilon)}$. Hence

$$
\left\|\left\{\Psi_{j, \pm}\right\}_{\mathbf{m}}\right\| \leq \frac{1}{\cos \theta_{ \pm}}\left\|\frac{\left\{F_{j, \pm}\right\}_{\mathbf{m}}(x, \epsilon)}{k \lambda(x, \epsilon)+2 x-\epsilon}\right\|
$$

with $\cos \theta_{ \pm}>\sin \eta>0, \eta$ as in (24).

Therefore

- for $\mathbf{m}=(n, n)+\mathbf{e}_{j}$ :

$$
\left\|\left\{\Psi_{j, \pm}\right\}_{(n, n)+\mathbf{e}_{j}}\right\| \leq c\left\|\left\{G_{j, \pm}\right\}_{(n, n)+\mathbf{e}_{j}}\right\|,
$$


- for a multi-index m with $m_{1}-m_{2}+(-1)^{j} \neq 0$ :

$$
\left\|\left\{\Psi_{j, \pm}\right\}_{\mathbf{m}}\right\| \leq c\left(\left\|\left\{\alpha_{ \pm}^{*} \Psi_{j, \pm}\right\}_{\mathbf{m}}\right\|+\left\|\left\{K_{ \pm} \cdot\left(v_{j}+\Psi_{j, \pm, \Delta}\right)\right\}_{\mathbf{m}}\right\|+\left\|\left\{G_{j, \pm}\right\}_{\mathbf{m}}\right\|\right)
$$

for some $c>0$.

Therefore the majorating series satisfies

$$
\begin{aligned}
\bar{\Psi}_{j, \pm} & \prec c \bar{\alpha}_{ \pm}^{*} \bar{\Psi}_{j, \pm}+c \bar{K}_{ \pm} \cdot\left(v_{j}+\bar{\Psi}_{j, \pm}\right)+c \bar{G}_{j, \pm} \\
& \prec 2 c\left(\bar{\chi}^{*} \circ \bar{\Phi}_{ \pm}\right) \cdot \bar{\Psi}_{j, \pm}+c \bar{k}_{ \pm} \cdot\left(v_{j}+\bar{\Psi}_{j, \pm}\right)+c \bar{f}_{j, \pm} \circ \bar{\Phi}_{ \pm} \\
& =: \bar{R}_{j, \pm}\left(v+\bar{\Psi}_{ \pm, \Delta}, \bar{\Psi}_{ \pm, \star}\right) \\
& \prec \bar{R}_{j, \pm}\left(v+\bar{\Psi}_{ \pm}, v+\bar{\Psi}_{ \pm}\right),
\end{aligned}
$$

where $K_{ \pm}=K_{ \pm}\left(v+\Psi_{ \pm, \Delta}, \Psi_{ \pm, \star}\right), \bar{k}_{ \pm}=\bar{K}_{ \pm}\left(v+\bar{\Psi}_{ \pm, \Delta}, \bar{\Psi}_{ \pm, \star}\right)$, and $\bar{R}_{j, \pm}\left(w_{1}, w_{2}\right)=$ $O\left(|w|^{2}\right)$, and we can conclude with Lemma 44.

The same thing is done also in the formal category with the Banach space $\hat{\mathfrak{B}}\left(\Omega_{ \pm}^{0}\right)$.

\subsection{Step 3: Final Reduction and Transverse Symplecticity of the Transformation}

Suppose that the system is in the form (71), and write

$$
\begin{aligned}
& \alpha_{ \pm}(h, x, \epsilon)=\tilde{\chi}_{ \pm}(h, x, \epsilon)+x(x-\epsilon) \beta_{ \pm}(h, x, \epsilon), \\
& \tilde{\chi}_{ \pm}(h, x, \epsilon)=\tilde{\chi}_{ \pm}^{(0)}(h, \epsilon)+x \tilde{\chi}_{ \pm}^{(1)}(h, \epsilon) .
\end{aligned}
$$

Then the transformation $v=e^{\int_{0}^{x} \beta_{ \pm} d x\left(\begin{array}{cc}1 & 0 \\ 0 & -1\end{array}\right)} u$ will bring it to the normal form with formal invariant $\tilde{\chi}_{ \pm}$.

Let $y=\Psi_{ \pm}(u, x, \epsilon)$ be the transformation obtained as a composition of the transformations of Steps 1-3, and $y=\hat{\Psi}(u, x, \epsilon)=\sum_{k, l} \psi^{(k, l)}(u) x^{k} \epsilon^{l}$ the corresponding formal transformation. By construction $\Psi_{ \pm}(\cdot, 0, \epsilon)=\hat{\Psi}(\cdot, 0, \epsilon)=\mathrm{id}$, in particular it is convergent. The restriction of $\Psi_{ \pm}(u, x, \epsilon)$ to $\mathrm{U} \times \Omega_{ \pm}$must agree with the Borel $(1,1)$-sum of $\hat{\Psi}(u, x, \epsilon)$ : indeed one can swap the summation in $u$ and of the Laplace integral by a dominated convergence argument.

Let us now show that $\Psi_{ \pm}(u, x, \epsilon)$ is transversely symplectic and therefore $\tilde{\chi}_{ \pm}=$ $\chi$. Let $u_{ \pm}(x, \epsilon ; c)$ be a germ of a general solution of the normal form system with the formal invariant equal to $\tilde{\chi}_{ \pm}$, depending on an initial condition parameter $c=$ $\left(c_{1}, c_{2}\right) \in\left(\mathbb{C}^{2}, 0\right), \operatorname{det}\left(D_{c} u_{ \pm}\right) \neq 0$, and let $y_{ \pm}(x, \epsilon ; c)=\Psi_{ \pm}\left(u_{ \pm}(x, \epsilon ; c), x, \epsilon\right)$ be the corresponding solution germ of the system (1). Then $D_{c} y_{ \pm}=D_{u} \Psi_{ \pm} \cdot D_{c} u_{ \pm}$ satisfies the linearized system

$$
x(x-\epsilon) \frac{d D_{c} y_{ \pm}}{d x}=J D_{y}^{2} H \cdot D_{c} y_{ \pm},
$$


and by the Liouville-Ostrogradskii formula

$$
x(x-\epsilon) \frac{d}{d x} \operatorname{det}\left(D_{c} y_{ \pm}\right)=\operatorname{tr}\left(J D_{y}^{2} H\right) \cdot \operatorname{det}\left(D_{c} y_{ \pm}\right),
$$

but $\operatorname{tr}\left(J D_{y}^{2} H\right)=0$, i.e. $\operatorname{det}\left(D_{c} y_{ \pm}\right)=\operatorname{det}\left(D_{u} \Psi_{ \pm}\right) \cdot \operatorname{det}\left(D_{c} u_{ \pm}\right)$is constant in $x$. Similarly, $\operatorname{det}\left(D_{c} u_{ \pm}\right)$is constant in $x$. Therefore $\operatorname{det}\left(D_{u} \Psi_{ \pm}(u, x, \epsilon)\right)$ is also constant in $x$, and equal to $\operatorname{det}\left(D_{u} \Psi_{ \pm}(u, 0, \epsilon)\right)=1$ since $\Psi_{ \pm}(u, 0, \epsilon)=u$.

This terminates the proof of Theorem 17.

\subsection{Proof of Proposition 9}

We will construct a formal symplectic change of coordinate $\Phi=\Phi\left(h, u_{i}\right)$, written as a formal power series in $h$ and $u_{i}$, such that $G=H \circ \Phi$. The transformation $\Phi$ is constructed recursively as a formal limit

$\Phi=\lim _{k \rightarrow+\infty} \Phi_{k, 1}, \quad \Phi_{k+1,1}=\lim _{l \rightarrow+\infty} \Phi_{k, l}, \quad \Phi_{0,1}=\mathrm{id}, \quad H \circ \Phi_{k, l}=G+O\left(h^{k} u_{i}^{l}\right)$.

At each step $(k, l), k \geq 0, l \geq 1$, we want to get rid of the power $h^{k} u_{i}^{l}$ in $H \circ \Phi_{k, l}$. We construct $\Phi_{k, l+1}=\Phi_{k, l} \circ \Phi_{f_{k, l} X_{h^{k} u_{i}^{l}}}$ as a composition of $\Phi_{k, l}$ with the time-1 flow of a Hamiltonian vector field $f_{k, l} X_{h^{k} u_{i}^{l}}=-(-1)^{i} f_{k, l} h^{k-1} u_{i}^{l}\left[k u_{i} \partial_{u_{i}}-(k+l) u_{j} \partial_{u_{j}}\right]$ for some $f_{k, l} \in \mathbb{C}$. The flow sends both $h$ and $u_{i}$ to functions of $\left(h, u_{i}\right)$,

$\Phi_{f_{k, l} X_{h^{k} u_{i}^{l}}}: \quad u_{i} \mapsto u_{i}+O\left(h^{k-1} u_{i}^{l+1}\right), \quad h \mapsto h+(-1)^{i} l f_{k, l} h^{k} u_{i}^{l}+O\left(h^{2 k-1} u_{i}^{2 l}\right)$

where the terms $O\left(h^{2 k-1} u_{i}^{2 l}\right)$ are null if $k=0$. If $H \circ \Phi_{k, l}=G+H_{k, l} h^{k} u_{i}^{l}+O\left(h^{k} u_{i}^{l+1}\right)$ for some $H_{k, l} \in \mathbb{C}$, then we want

$$
\begin{aligned}
\left(G+H_{k, l} h^{k} u_{i}^{l}\right) \circ \Phi_{f_{k, l} X_{h^{k} u_{i}^{l}}^{1}}-G(h) & =O\left(h^{k} u_{i}^{l+1}\right), \\
H_{k, l} h^{k} u_{i}^{l}+\lambda \cdot(-1)^{i} l f_{k, l} h^{k} u_{i}^{l} & =O\left(h^{k} u_{i}^{l+1}\right), \\
f_{k, l} & =-(-1)^{i} \frac{H_{k, l}}{\lambda l} .
\end{aligned}
$$

Now that we have constructed the formal symplectic transformation $\Phi$, we can conclude by the following Proposition.

Proposition 49 Let $H, \tilde{H}:\left(\mathbb{C}^{2}, 0\right) \rightarrow(\mathbb{C}, 0)$ be two germs with a non-degenerate critical point at 0 , and let $\omega, \tilde{\omega}$ be germs of symplectic forms. Then the two pairs $(H, \omega),(\tilde{H}, \tilde{\omega})$ are analytically equivalent if and only if they are formally equivalent.

Proof By Theorem 4, the Birkhoff-Siegel normal form is, up to the involution (13), a complete analytic invariant for each pair. Therefore it is enough to show that it is 
also a formal invariant. This can be seen from the invariance of a formalization of the formula (15) of Sect. 2.2.1.

Acknowledgements Open access funding provided by University of Vienna. This paper was largely inspired by the works of C. Rousseau and L. Teyssier [25], C. Lambert and C. Rousseau [15], and A. Bittmann $[3,4]$. It was written during my stay at Centre de Recherches Mathématiques at Université de Montréal. I want to thank Christiane Rousseau for her kind support and the CRM for its hospitality.

Open Access This article is distributed under the terms of the Creative Commons Attribution 4.0 International License (http://creativecommons.org/licenses/by/4.0/), which permits unrestricted use, distribution, and reproduction in any medium, provided you give appropriate credit to the original author(s) and the source, provide a link to the Creative Commons license, and indicate if changes were made.

\section{References}

1. Arnold, V.I., Varchenko, A.N., Gusein-Zade, S.M.: Singularities of Differentiable Maps, Volume II: Monodromy and Asymptotics of Integrals. Birkhäuser, Boston (2012)

2. Balser, W.: Summability of power series in several variables, with applications to singular perturbation problems and partial differential equations Ann. Fac. Sci. Toulouse Math. 14, 593-608 (2005)

3. Bittmann, A.: Doubly-resonant saddle-nodes in $\left(\mathbb{C}^{3}, 0\right)$ and the fixed singularity at infinity in the Painlevé equations: formal classification. Qual. Theory Dyn. Syst. 16, 491-529 (2016)

4. Bittmann, A.: Doubly-resonant saddle-nodes in $\left(\mathbb{C}^{3}, 0\right)$ and the fixed singularity at infinity in the Painlevé equations: Analytic classification. Ann. Inst. Fourier (2017). arXiv:1709.10333 (to appear)

5. Bonckaert, P., De Maesschalck, P.: Gevrey normal forms of vector fields with one zero eigenvalue. J. Math. Anal. Appl. 344, 301-321 (2008)

6. Costin, O., Tanveer, S.: Existence and uniqueness for a class of nonlinear higher-order partial differential equations in the complex plane. Comm. Pure Appl. Math. 8, 0001-0026 (2000)

7. Duval, A.: Confluence procedures in the generalized hypergeometric family. J. Math. Sci. Univ. Tokyo 5, 597-625 (1998)

8. Françoise, J.-P., Smaiili, M.: Lemme de Morse transverse pour des puissances de formes de volume. Ann. Fac. Sci. Toulouse 6e Série 3, 81-89 (1994)

9. Glutsyuk, A.: Confluence of singular points and the nonlinear Stokes phenomena. Trans. Mosc. Math. Soc. 62, 49-95 (2001)

10. Hurtubise, J., Lambert, C., Rousseau, C.: Complete system of analytic invariants for unfolded differential linear systems with an irregular singularity of Poincaré rank k. Mosc. Math. J. 14, 309-338 (2013)

11. Ilyashenko, Y., Yakovenko, S.: Lectures on Analytic Differential Equations, Grad. Studies Math. 86, Amer. Math. Soc., Providence (2008)

12. Kawai, T., Takei, Y.: Algebraic Analysis of Singular Perturbation Theory. Translations of Mathematical Monographs, vol. 277. American Mathematical Society, Providence (2005)

13. Klimeš, M.: Confluence of singularities of non-linear differential equations via Borel-Laplace transformations. J. Dyn. Control Syst. 22, 285-324 (2016)

14. Klimeš, M.: Non-linear Stokes phenomenon in the fifth Painlevé equation and a wild monodromy action on the character variety, arXiv:1609.05185 (2017)

15. Lambert, C., Rousseau, C.: Complete system of analytic invariants for unfolded differential linear systems with an irregular singularity of Poincaré rank 1. Mosc. Math. J. 12, 77-138 (2012)

16. Mardešić, P., Roussarie, R., Rousseau, C.: Modulus of analytic classification for unfoldings of generic parabolic diffeomorphisms. Mosc. Math. J. 4, 455-502 (2004)

17. Martinet, J., Ramis, J.-P.: Problèmes de modules pour des équations différentielles non linéaires du premier ordre. Publ. Math. IHES 55, 63-164 (1982)

18. Martinet, J., Ramis, J.-P.: Classification analytique des équations différentielles non linéaires résonnantes du premier ordre. Ann. Sci. É.N.S. 4e série 16, 571-621 (1983)

19. Martinet, J., Ramis, J.-P.: Elementary acceleration and multisummability. I. Ann. Inst. Henri Poincaré (A) Physique théorique 54, 331-401 (1991) 
20. Mattei, J.-F., Moussu, R.: Holonomie et intégrales premières. Ann. Sci. É.N.S. 4e série 13, 469-523 (1980)

21. Okamoto, K.: Polynomial Hamiltonians associated with Painlevé Equations. I. Proc. Jpn. Acad. Ser. A Math. Sci. 56, 264-268 (1980)

22. Parise, L.: Confluence de singularités régulières d'équations différentielles en une singularité irrégulière. Modèle de Garnier, thèse de doctorat, IRMA Strasbourg (2001). [http://irma.math.unistra. fr/annexes/publications/pdf/01020.pdf]

23. Ramis, J.-P.: Confluence and resurgence. J. Fac. Sci. Univ. Tokyo Sec. IA 36, 703-716 (1989)

24. Rebelo, J., Reis, H.: Local theory of holomorphic foliations and vector fields, arXiv:1101.4309 (2011)

25. Rousseau, C., Teyssier, L.: Analytical moduli for unfoldings of saddle-node vector fields. Mosc. Math. J. 8, 547-614 (2008)

26. Rousseau, C., Teyssier, L.: Analytical normal and inverse problems forms for unfoldings of 2dimensional saddle-nodes with analytic center manifold, hal-01301236v2. Ann. Sci. É.N.S. (2017) (to appear)

27. Shimomura, S.: Analytic integration of some nonlinear ordinary differential equations and the fifth Painlevé equation in the neighborhood of an irregular singular point. Funkcialaj Ekvacioj 26, 301-338 (1983)

28. Siegel, C.L., Moser, J.K.: Lectures on Celestial Mechanics. Grundlehren der mathematische Wissenschaften, vol. 187. Springer, Berlin (1971)

29. Singer, M., van der Put, M.: Galois Theory of Linear Differential Equations. Grundlehren der Mathematische Wissenschaften, vol. 328. Springer, Berlin (2003)

30. Takano, K.: A 2-parameter family of solutions of Painlevé equation (V) near the point at infinity. Funkcialaj Ekvacioj 26, 79-113 (1983)

31. Takano, K.: Reduction for Painlevé equations at the fixed singular points of the first kind. Funkcialaj Ekvacioj 29, 99-119 (1986)

32. Takano, K.: Reduction for Painlevé equations at the fixed singular points of the second kind. J. Math. Soc. Jpn. 42, 423-443 (1990)

33. Teyssier, L.: Équation homologique et cycles asymptotiques d'une singularité col-nœud. Bull. Sci. Math. 128, 167-187 (2004)

34. Yoshida, S.: A general solution of a nonlinear 2-system without Poincaré's condition at an irregular singular point. Funkcialaj Ekvacioj 27, 367-391 (1984)

35. Yoshida, S.: 2-parameter family of solutions of Painlevé equations (I)-(V) at an irregular singular point. Funkcialaj Ekvacioj 28, 233-248 (1985)

36. Vey, J.: Sur le lemme de Morse. Invent. Math. 40, 1-9 (1977) 